\title{
Distributed Bregman-Distance Algorithms for Min-Max Optimization
}

\author{
Kunal Srivastava, Angelia Nedić and Dušan Stipanović
}

\begin{abstract}
We consider a min-max optimization problem over a time-varying network of computational agents, where each agent in the network has its local convex cost function which is a private knowledge of the agent. The agents want to jointly minimize the maximum cost incurred by any agent in the network, while maintaining the privacy of their objective functions. To solve the problem, we consider subgradient algorithms where each agent computes its own estimates of an optimal point based on its own cost function, and it communicates these estimates to its neighbors in the network. The algorithms employ techniques from convex optimization, stochastic approximation and averaging protocols (typically used to ensure a proper information diffusion over a network), which allow time-varying network structure. We discuss two algorithms, one based on exact-penalty approach and the other based on primal-dual Lagrangian approach, where both approaches utilize Bregman-distance functions. We establish convergence of the algorithms (with probability one) for a diminishing step-size, and demonstrate the applicability of the algorithms by considering a power allocation problem in a cellular network.
\end{abstract}

\section{Introduction}

This work is motivated by coordinator-free distributed algorithms for optimization problems originating in $[38,5]$, which have recently seen a resurgence driven by wireless network applications. A canonical problem in coordinator-free distributed setting is the problem of reaching an agreement among the local decision variables

Kunal Srivastava

University of Illinois, Urbana, IL 61801, USA, e-mail: kkunal2@illinois.edu

Angelia Nedić

University of Illinois, Urbana, IL 61801, USA, e-mail: angelia@illinois.edu

Dušan Stipanović

University of Illinois, Urbana, IL 61801, USA, e-mail: dusan@illinois.edu 
in a network of computational agents [39]. Protocols for achieving an agreement employ local averaging which is known to be robust to time-varying graph topology and noisy communication links [18]. These averaging protocols have led to a new class of distributed algorithms for parameter estimation [37], distributed optimization $[29,25,23,19,21,35]$ and control of multi-agent systems [16]. Recent work in agreement-based distributed optimization has focused on minimizing the sum of agents' local cost functions [25, 23, 35, 17, 19, 20,32], which arises in wide variety of areas ranging from sensor networks [28] to distributed machine learning [1].

Optimizing the sum of local objective functions is a popular choice for problems arising in resource allocation and network utility maximization [34], as the objective function is a measure of fair resource allocation. An alternative notion of fairness is the min-max criterion [8], where the interest is in determining an optimal decision variable that minimizes the worst case loss incurred by any agent. However, the algorithms for solving distributed min-max problems over networks are in their infancy. To fill out this void, we have recently considered a distributed method for solving such a problem in [36], where we provided a subgradient algorithm based on the exact-penalty function approach. Here, we make a further progress in two different directions: (1) we present a distributed exact penalty algorithm that uses Bregman distances [10] as opposed to the standard Euclidean distance considered in [36]; and (2) we provide an alternative distributed primal-dual algorithm that also uses Bregman distances. We establish convergence of both algorithms for a diminishing step-size and time-varying network, under mild conditions on the network connectivity. We allow for stochastic errors in subgradient evaluations, which are assumed to be zero-mean and with uniformly bounded expected norm, as typically done in stochastic approximations or stochastic subgradient methods [13, 14, 27, 6, 9, 26].

The min-max optimization aspect addressed in this paper is novel with respect to the prior work on distributed optimization over a network, which is dealing exclusively with the minimization of the sum of agent objectives $[29,25,23,19,21,1,35$, 32], except for [36]. The network aspect of this paper sets it apart from the standard optimization problems with noisy (sub)-gradient evaluations [14, 27, 6, 9, 26].

The advantage of the proposed algorithms is their ability to solve a class minmax distributed problems for which currently there are no specific algorithms, except for [36]. The difficulty in developing such algorithms comes from the inherent distributed knowledge of the problem data in a network of agents. Thus, there is a need for an algorithm that has ability to "align" the iterates among the agents while simultaneously solving the network problem. Both of our proposed algorithms achieve this task.

Regarding the benefits of the proposed algorithms, the exact-penalty algorithm is simpler for implementation, but in principle it is a distributed subgradient approach due to the use of non-differentiable penalty functions. The primal-dual approach requires a larger number of variables than the penalty approach, but it preserves the smoothness of the problem (provided that the original agent objective functions $f_{i}$ are smooth). In the presence of stochastic errors in (sub)gradient evaluations, both algorithms will have overall rate of the order of $1 / \sqrt{k}$ in terms of the number of iterations $k$, which is typical for stochastic approximations [27, 26]. 
The rest of the chapter is organized as follows. In Section 2 we state our problem of interest and suitably reformulate it for the development of distributed algorithms. In Section 3 we present our distributed Bregman-distance based algorithm which utilizes the exact penalty function approach, and we establish the convergence of the algorithm. In Section 4 we develop an alternative algorithm which builds on the primal-dual approach of Arrow-Hurwicz-Uzawa [2], and we prove its convergence. This algorithm paves a way to handling the problems in which the network plays a min-max game against an exogenous signal, which is discussed in Section 5. In Section 6 we present an example of min-max power allocation in a cellular network and provide simulation results for both the exact penalty approach and the primaldual approach. We conclude in Section 7.

Notation: The set of real numbers is denoted by $\mathbb{R}$, while non-negative real numbers are denoted by $\mathbb{R}_{+}$. All vectors are viewed as columns, where the $j^{\text {th }}$ component of a vector $x$ is denoted by $x_{j}$. We use the symbol $\langle x, y\rangle$ to denote the inner-product between two vectors $x$ and $y$. We write 1 for the vector with each component equal to 1 . A vector $\pi$ is stochastic if $\pi_{i} \geq 0$ for all $i$ and $\sum_{i} \pi_{i}=1$.

For an $m \times m$ matrix $A$, we use $A_{i j}$ or $[A]_{i j}$ to denote its entry in the $i^{\text {th }}$ row and $j^{\text {th }}$ column. An $m \times m$ matrix $W$ is stochastic if $W_{i j} \geq 0$ for all $i, j$, and $W \mathbf{1}=\mathbf{1}$. A stochastic matrix $W$ is doubly stochastic if it satisfies $\mathbf{1}^{T} W=\mathbf{1}$. Given a directed graph $G=(V, E)$, the link $(i, j) \in E$ is to be interpreted as the incoming edge from $j$ to $i$. For a bidirectional graph $G$, we have $(i, j) \in E$ if and only if $(j, i) \in E$. We will sometimes denote the edge set of a graph $G$ as $\mathscr{E}(G)$. We use the terms "agent" and "node" interchangeably. We say that agent $j$ is a neighbor of agent $i$ if $(i, j) \in E$, and we denote the set of all neighbors of agent $i$ by $N_{i}$. When the edge set is time varying, we use $N_{i}(t)$ to denote the neighbors of agent $i$ at time $t$. Given a logical statement $p(x)$ that is predicated on a variable $x$, we use $\mathbf{1}_{\{p(x)\}}$ to denote the indicator function which takes value 1 when $p(x)$ is true and value 0 when $p(x)$ is false.

\section{Problem Formulation}

We consider a system of $m$ computational agents, which is viewed as the node set $V=\{1, \ldots, m\}$. We assume that the time is discrete and use $k=0,1, \ldots$ to denote the time instances. The agents communicate with each other over a time-varying communication network. At any time $k$, the communications among the agents are represented by a directed graph $G(k)=(V, E(k))$ with an edge-set $E(k)$ that has a link $(i, j) \in E(k)$ if and only if agent $i$ receives information from agent $j$ at time $k$.

Let each agent $i$ have a cost function $f_{i}$, which is known only to that agent. Consider a distributed multi-agent optimization problem subject to local agent communications, where the agents want to cooperatively solve the following problem:

$$
\min _{x \in X} \max _{i \in V} f_{i}(x) .
$$


Here, each $f_{i}: \mathbb{R}^{n} \rightarrow \mathbb{R}$ is a convex function, representing a local objective function known only by agent $i$. The set $X \subseteq \mathbb{R}^{n}$ is a closed and convex set known by all agents. Throughout the paper, we assume that the problem has a nonempty optimal set, which is denoted by $X^{*}$.

We assume that $\mathbb{R}^{n}$ is equipped with some norm $\|\cdot\|$, with a corresponding dual norm $\|\cdot\|_{*}$. The goal is to develop a distributed algorithm for solving the constrained optimization problem in (1), while obeying the network connectivity structure and local information exchange among the neighboring agents.

The min-max-problem in (1) is a convex problem, as the function $f(x)=$ $\max _{i \in V} f_{i}(x)$ is convex since point-wise maximum of convex functions preserves convexity ([4], Proposition 1.2.4, page 30). We are interested in the case when the agents' objective functions $f_{i}$ are not necessarily differentiable. We also allow the local objective functions $f_{i}$ to take the form of the following stochastic optimization:

$$
f_{i}(x)=\mathbb{E}_{\omega_{i}}\left[F_{i}\left(x, \omega_{i}\right)\right]+\Omega_{i}(x),
$$

where the expectation is taken with respect to the distribution of a random variable $\omega_{i}$. The term $\Omega_{i}(x)$ is a regularization term that may be included to improve the generalization ability [15], or to enforce sparsity on solutions.

Min-max problem (1) does not lend itself to distributed computations that obey the local connectivity of the agents in the network. We find it useful to use the epigraph representation of the problem. In particular, we let $\eta \in \mathbb{R}$ and we re-cast problem (1) in an equivalent form:

$$
\begin{array}{ll}
\operatorname{minimize} & \eta \\
\text { subject to } & f_{i}(x) \leq \eta \quad \text { for all } x \in X, \eta \in \mathbb{R}, \text { and } i \in V,
\end{array}
$$

where $x$ and $\eta$ are variables. We use $\eta^{*}$ to denote the optimal value of problem (3).

To distributedly solve the min-max problem, we provide two algorithms aimed at solving its epigraph formulation in (3). The first algorithm is based on an exact penalty function approach and the second algorithm is based on a primal-dual approach, where both algorithms employ Bregman-distance functions.

Before proceeding with the algorithmic development, we provide some basics of a Bregman-distance function as introduced by Bregman [10] (also, see for example [11]). Let $\mathbb{R}^{n}$ be equipped with some norm $\|\cdot\|$, whose dual norm is $\|x\|_{*}=\sup _{\|y\| \leq 1}\langle y, x\rangle$. Let $X \subseteq \mathbb{R}^{n}$ be a convex set and $\omega: X \rightarrow \mathbb{R}$ be a differentiable convex function over $X$. The function $\omega$ is strongly convex with a parameter $\sigma>0$ (with respect to the norm $\|\cdot\|$ ), if it satisfies

$$
\omega(y)-[\omega(x)+\langle\nabla \omega(x), y-x\rangle] \geq \frac{\sigma}{2}\|x-y\|^{2} \quad \text { for all } x \in X^{\circ} \text { and } y \in X,
$$

where $X^{\circ}$ denotes the relative interior of the set $X$. Alternatively, $\omega$ is strongly convex over $X$ with a parameter $\sigma>0$ if there holds:

$$
\langle\nabla \omega(x)-\nabla \omega(y), x-y\rangle \geq \sigma\|x-y\|^{2} \quad \text { for all } x, y \in X^{\circ} .
$$


Given a strongly convex function $\omega$, we can define the Bregman-distance function $B: X \times X^{\circ} \rightarrow \mathbb{R}_{+}$induced by $\omega:$

$$
B(y, x)=\omega(y)-[\omega(x)+\langle\nabla \omega(x), y-x\rangle] .
$$

This function is also referred to as the prox-function induced by $\omega$. For the function $B$, by the convexity of $\omega$ we have

$$
B(y, x) \geq 0 \quad \text { for all } y \in X \text { and } x \in X^{\circ} .
$$

Furthermore, by the strong convexity of $\omega$, for every $x \in X^{\circ}$, the function $B(\cdot, x)$ is strongly convex over $X$ with the same parameter $\sigma$. The Bregman function $B(y, x)$ is used to define a nonlinear projection operator associated with the given set $X$, also known as the prox-operator [26], as follows:

$$
P_{X}(d, x)=\operatorname{argmin}_{z \in X}\{\langle d, z-x\rangle+B(z, x)\} .
$$

\section{Exact Penalty Function Approach}

We further transform the problem in (3) by penalizing the constraints to obtain the following problem:

$$
\min _{x \in X, \eta \in \mathbb{R}} \eta+\sum_{i=1}^{m} r_{i} g_{i}(x, \eta)
$$

where each $g_{i}$ is a penalty function given by

$$
g_{i}(x, \eta)=\max \left\{0, f_{i}(x)-\eta\right\}
$$

and $r_{i}>0$ is a penalty parameter for violating the constraint $f_{i}(x) \leq \eta$. Under certain conditions [3], the solutions of the penalized problem (4) are also the solutions of the constrained problem (3). Specifically, these conditions involve the Lagrangian dual problem associated with problem (3). We introduce the Lagrangian function:

$$
L(x, \eta, \mu)=\eta+\sum_{i=1}^{m} \mu_{i}\left(f_{i}(x)-\eta\right)
$$

where $\mu=\left(\mu_{1}, \ldots, \mu_{m}\right)^{\prime}$ is the vector of dual variables satisfying $\mu_{i} \geq 0$ for all $i \in V$. The dual problem is

$$
\max _{\mu \geq 0} q(\mu) \quad \text { with } \quad q(\mu)=\inf _{x \in X, \eta \in \mathbb{R}} L(x, \eta, \mu) .
$$

It can be verified that the Slater condition is satisfied for problem (3) and, hence, there is no duality gap between the primal problem (3) and its dual (6). Furthermore, the set of dual optimal solutions is nonempty and bounded. The bound for the dual optimal variables can be found by rewriting the Lagrangian function (5) as follows: 


$$
L(x, \eta, \mu)=\left(1-\sum_{i=1}^{m} \mu_{i}\right) \eta+\sum_{i=1}^{m} \mu_{i} f_{i}(x) .
$$

Thus, $\inf _{\eta \in \mathbb{R}} L(x, \eta, \mu)=-\infty$ when $\sum_{i=1}^{m} \mu_{i} \neq 1$, implying that $q(\mu)=-\infty$ whenever $\sum_{i=1}^{m} \mu_{i} \neq 1$. Therefore, the domain of the dual function $q$ is the set of multipliers $\mu \geq 0$ such that $\sum_{i=1}^{m} \mu_{i}=1$, showing that optimal multipliers $\mu_{i}^{*}$ must satisfy

$$
\sum_{i=1}^{m} \mu_{i}^{*}=1
$$

Hence, according to [3], when the penalty parameters satisfy $r_{i}>1$ for all $i$, then the problems in (4) and (3) are equivalent.

Penalized problem (4) has a form suitable for distributed computations among the agents, as its objective can be written as $\sum_{i=1}^{m}\left(\eta / m+r_{i} g_{i}(x, \eta)\right)$ and the function $\tilde{F}_{i}(x, \eta)=\eta / m+r_{i} g_{i}(x, \eta)$ can be interpreted as an objective function associated with agent $i$. In this setting, agent $i$ is the only agent that knows the function $f_{i}$ and, therefore, this agent is the only agent that deals with the dual variable $\mu_{i}$ associated with the constraint $f_{i}(x) \leq \eta$. Furthermore, observe that there is no need for any coordination of the penalty values $r_{i}$ among the agents, as each agent just needs to choose its individual penalty $r_{i}>1$.

\subsection{Equivalence Between Epigraph and Penalty Formulations}

We establish an important relation between the optimal solutions of the epigraph formulation (3) of the min-max problem and its penalized counterpart (4). The proof of this relation is basically along the lines of the work in [3], but somewhat shorter as it exploits the special structure of the epigraph formulation (3). Furthermore, the relation is important in our further development and it is not readily available.

To prove the result, we use the saddle-point theorem characterizing the optimal solutions of problem (3) and its dual problem (6), as given for example in [4], Proposition 6.2.4, page 360. The theorem is adjusted to the specific form of our Lagrangian function.

Theorem 1. The pair $\left(z^{*}, \mu^{*}\right)$ with $z^{*}=\left(x^{*}, \eta^{*}\right) \in X \times \mathbb{R}$ and $\mu^{*} \geq 0$ is a saddlepoint of the Lagrangian function $L(z, \mu)$ (i.e., primal-dual optimal pair) if and only if the following relation holds:

$$
L\left(z^{*}, \mu\right) \leq L\left(z^{*}, \mu^{*}\right) \leq L\left(z, \mu^{*}\right) \quad \text { for all } z=(x, \eta) \in X \times \mathbb{R} \text { and } \mu \geq 0 .
$$

Now, we have the following lemma.

Lemma 1. Let $\eta^{*}=\min _{x \in X} \max _{i \in V} f_{i}(x)$ and $r_{i}>1$ for all $i$. Then, for $g_{i}(x, \eta)=$ $\max \left\{0, f_{i}(x)-\eta\right\}$ we have 


$$
\sum_{i=1}^{m} r_{i} g_{i}(x, \eta)+\eta \geq \eta^{*} \quad \text { for all } x \in X \text { and } \eta \in \mathbb{R} .
$$

Furthermore, the above inequality holds as equality if and only if $\eta=\eta^{*}$ and $x=x^{*}$ for an optimal solution $x^{*}$ of the problem $\min _{x \in X} \max _{i} f_{i}(x)$.

Proof. Consider the definition of the Lagrangian in (5). For a given $x \in X$ and $\eta$, let us define the dual variables $\mu_{i}$ such that $\mu_{i}=r_{i}$ if $f_{i}(x)-\eta \geq 0$, and $\mu_{i}=0$ if $f_{i}(x)-\eta<0$, or compactly $\mu_{i}=r_{i} \mathbf{1}_{\left\{f_{i}(x) \geq \eta\right\}}$. Then, we have

$$
\sum_{i=1}^{m} r_{i} \max \left\{f_{i}(x)-\eta, 0\right\}+\eta=\sum_{i=1}^{m} \mu_{i}\left(f_{i}(x)-\eta\right)+\eta=L(z, \mu) .
$$

Furthermore, we have $L\left(z^{*}, \mu^{*}\right)=\eta^{*}$. Thus, we need to prove that $L(z, \mu)-$ $L\left(z^{*}, \mu^{*}\right) \geq 0$. For this we use Theorem 1 , from which we obtain $-L\left(z^{*}, \mu^{*}\right) \geq$ $-L\left(z, \mu^{*}\right)$, implying

$$
\begin{aligned}
L(z, \mu)- & L\left(z^{*}, \mu^{*}\right) \geq L(z, \mu)-L\left(z, \mu^{*}\right)=\sum_{i=1}^{m}\left(\mu_{i}-\mu_{i}^{*}\right)\left(f_{i}(x)-\eta\right) \\
& =\sum_{i=1}^{m}\left(r_{i}-\mu_{i}^{*}\right) \mathbf{1}_{\left\{f_{i}(x) \geq \eta\right\}}\left(f_{i}(x)-\eta\right)-\sum_{i=1}^{m} \mu_{i}^{*} \mathbf{1}_{\left\{f_{i}(x)<\eta\right\}}\left(f_{i}(x)-\eta\right) \geq 0,
\end{aligned}
$$

where we have used the decomposition $\mu_{i}^{*}=\mu_{i}^{*} \mathbf{1}_{\left\{f_{i}(x) \geq \eta\right\}}+\mu_{i}^{*} \mathbf{1}_{\left\{f_{i}(x)<\eta\right\}}$, and relations $r_{i}>1$ and $1 \geq \mu_{i}^{*} \geq 0$ for all $i$ (see (7)).

We next show that the preceding inequality holds as equality if and only if $\eta=\eta^{*}$ and $x=x^{*}$ for some $x^{*} \in X^{*}$. By the definition of min-max solution, if $x^{*}$ solves the problem then we have $f_{i}\left(x^{*}\right) \leq \eta^{*}$ for all $i$, implying

$$
\sum_{i=1}^{m} r_{i} \max \left\{f_{i}\left(x^{*}\right)-\eta^{*}, 0\right\}+\eta^{*}=\eta^{*} .
$$

Thus, we just need to prove the "only if" part. Assume that for some $\bar{x} \in X$ and $\bar{\eta}$,

$$
\sum_{i=1}^{m} r_{i} \max \left\{f_{i}(\bar{x})-\bar{\eta}, 0\right\}+\bar{\eta}=\eta^{*} .
$$

Since $\sum_{i=1}^{m} r_{i} \max \left\{f_{i}(\bar{x})-\bar{\eta}, 0\right\} \geq 0$, it follows $\bar{\eta} \leq \eta^{*}$. Let us assume that $\bar{\eta}<\eta^{*}$. Then, for the equality to hold we must have $f_{j}(\bar{x})>\bar{\eta}$ for some $j$. Thus, $f_{i^{*}}(\bar{x})>\bar{\eta}$ for $i^{*}=\operatorname{argmax}_{i} f_{i}(\bar{x})$. By $\eta^{*}=\min _{x \in X} \max _{i \in V} f_{i}(x)$ we have $f_{i^{*}}(\bar{x}) \geq \eta^{*}$ implying $f_{i^{*}}(\bar{x})-\bar{\eta} \geq \eta^{*}-\bar{\eta}>0$. Since $r_{i^{*}}>1$, it follows that $r_{i^{*}}\left(f_{i^{*}}(\bar{x})-\bar{\eta}\right)>\eta^{*}-\bar{\eta}$. Therefore,

$$
\sum_{i=1}^{m} r_{i} \max \left\{f_{i}(\bar{x})-\bar{\eta}, 0\right\}+\bar{\eta}>\eta^{*}
$$


which contradicts (8). Hence, we must have $\bar{\eta}=\eta^{*}$ in (8). This further yields $\sum_{i=1}^{m} r_{i} \max \left\{f_{i}(\bar{x})-\eta^{*}, 0\right\}=0$, which by $r_{i}>0$ implies $f_{i}(\bar{x}) \leq \eta^{*}$ for all $i$, thus showing that $\bar{x}$ is a min-max solution.

\subsection{Penalty-Based Algorithm}

Here, we present a distributed multi-agent algorithm for solving the penalty reformulation (4) of the min-max problem, where a penalty function $g_{i}(x, \eta)$ is associated with an agent $i$ and $r_{i}>1$ for all $i \in V$. Let $x_{k}^{j}$ and $\eta_{k}^{j}$ be the decision variables of agent $j$ at time $k$, which are agent $j$ estimates of an optimal solution $x^{*}$ and the optimal value $\eta^{*}$ of the problem, respectively. Recall that the agents' communications at time $k$ are represented with a graph $G(k)=(V, E(k))$, where $(i, j) \in E(k)$ if agent $i$ receives estimates $x^{j}(k)$ and $\eta^{j}(k)$ from agent $j$. To capture this information exchange, we let $N_{i}(k)$ denote the set of neighbors of agent $i$, i.e., $N_{i}(k)=\{j \in V \mid(i, j) \in E(k)\}$. Let us introduce a strongly convex function $\omega_{x}: X \rightarrow \mathbb{R}$, with a parameter $\sigma_{x}>0$. We assume that $\mathbb{R}$ is equipped with square norm, i.e., $r \mapsto \frac{1}{2} r^{2}$, and we introduce a scalar function $\omega_{\eta}: \mathbb{R} \rightarrow \mathbb{R}$ that is strongly convex with respect to this norm, with a parameter $\sigma_{\eta}>0$. Let us denote the Bregman-distance functions generated by these strongly convex functions by $B_{x}(\cdot, \cdot)$, and $B_{\eta}(\cdot, \cdot)$ respectively, i.e.,

$$
\begin{aligned}
& B_{x}(y, u)=\omega_{x}(y)-\left[\omega_{x}(u)+\left\langle\nabla \omega_{x}(u), y-u\right\rangle\right], \\
& B_{\eta}(\phi, \zeta)=\omega_{\eta}(\phi)-\left[\omega_{\eta}(\zeta)+\omega_{\eta}^{\prime}(\zeta)(\phi-\zeta)\right],
\end{aligned}
$$

where $\omega_{\eta}^{\prime}(\zeta)$ denotes the derivative of $\omega_{\eta}$ at $\zeta$. Upon receiving the estimates $x_{k}^{j}$ and $\eta_{k}^{j}$ from its neighbors, each agent $i$ performs an intermittent adjustment of its estimates as follows:

$$
\left[\begin{array}{c}
\tilde{x}_{k}^{i} \\
\tilde{\eta}_{k}^{i}
\end{array}\right]=\sum_{j \in N_{i}(k)} w_{i j}(k)\left[\begin{array}{l}
x_{k}^{j} \\
\eta_{k}^{j}
\end{array}\right],
$$

where $w_{i j}(k) \geq 0$ is a weight that agent $i$ assigns to its neighbor $j \in N_{i}(k)$.

For a compact representation of relation (9), let $w_{i j}(k)=0$ for all $j \notin N_{i}(k)$ and introduce a matrix $W_{k}$ with entries $w_{i j}(k)$. With this notation, the intermittent adjustment in (9) can be written as follows:

$$
\tilde{z}_{k}^{i}=\left[\begin{array}{c}
\tilde{x}_{k}^{i} \\
\tilde{\eta}_{k}^{i}
\end{array}\right]=\sum_{j=1}^{m}\left[W_{k}\right]_{i j}\left[\begin{array}{c}
x_{k}^{j} \\
\eta_{k}^{j}
\end{array}\right] .
$$

After the intermittent adjustment, each agent $i$ takes a step toward minimizing its own penalty function through an adjustment of the following form: for all $i \in V$, 


$$
\begin{aligned}
& x_{k+1}^{i}=\operatorname{argmin}_{y \in X}\left[\alpha_{k} r_{i}\left\langle\nabla_{x} g_{i}\left(\tilde{z}_{k}^{i}\right)+\varepsilon_{k}^{i}, y\right\rangle+B_{x}\left(y, \tilde{x}_{k}^{i}\right)\right], \\
& \eta_{k+1}^{i}=\operatorname{argmin}_{s \in \mathbb{R}}\left[\alpha_{k}\left(\frac{1}{m}+r_{i} \nabla_{\eta} g_{i}\left(\tilde{z}_{k}^{i}\right)\right) s+B_{\eta}\left(s, \tilde{\eta}_{k}^{i}\right)\right],
\end{aligned}
$$

where $r_{i}>1$ and $\alpha_{k}>0$ is a step size. The notation $\nabla_{x} g_{i}(x, \eta)$ denotes a subgradient of $g$ with respect to $x$, i.e., the term $\nabla f_{i}(x) \mathbf{1}_{\left\{f_{i}(x) \geq \eta\right\}}$ where we use $\nabla f_{i}(x)$ to denote a subgradient of $f_{i}$ at $x$. Similarly, $\nabla_{\eta} g_{i}(x, \eta)$ denotes the partial derivative of $g$ with respect to $\eta$ i.e., $\nabla_{\eta} g_{i}(x, \eta)=-\mathbf{1}_{\left\{f_{i}(x) \geq \eta\right\}}$.

If $\mathbb{R}^{n}$ is equipped with the Euclidean norm, and the Bregman distance functions are chosen as $\omega_{x}(y)=\frac{1}{2}\|y\|^{2}$ and $\omega_{\eta}(\zeta)=\frac{1}{2} \zeta^{2}$, then algorithm (10)-(11) reduces to the standard subgradient-projection method:

$$
\left[\begin{array}{l}
x_{k+1}^{i} \\
\eta_{k+1}^{i}
\end{array}\right]=\Pi_{X \times \mathbb{R}}\left[\tilde{z}_{k}^{i}-\alpha_{k} r_{i}\left(\nabla g_{i}\left(\tilde{z}_{k}^{i}\right)+\left[\begin{array}{c}
\varepsilon_{k}^{i} \\
0
\end{array}\right]\right)\right],
$$

where $\Pi_{K}$ stands for the Euclidean projection on a set $K$.

Let us now take a closer look at the first update relation in (11). This update involves taking a step along an erroneous subgradient of $g_{i}$ at point $\tilde{z}_{k}^{i}$, i.e., the direction $\nabla_{x} g_{i}\left(\tilde{z}_{k}^{i}\right)+\varepsilon_{k}^{i}$ where $\varepsilon_{k}^{i}$ is a subgradient error. The agent $i$ objective function $g_{i}(x, \eta)=\max \left\{f_{i}(x)-\eta, 0\right\}$ is not differentiable at the point $(x, \eta)$ where $f_{i}(x)-$ $\eta=0$. At such a point, a subgradient of the function $g_{i}$ at $(x, \eta)$ exists since each function $f_{i}$ is assumed to be convex over the entire space ([4], Proposition 4.2.1). A subgradient of $g$ at such a point is given by

$$
\nabla g_{i}(x, \eta)=\left[\begin{array}{c}
\nabla f_{i}(x) \\
-1
\end{array}\right] \mathbf{1}_{\left\{f_{i}(x) \geq \eta\right\}}
$$

where $\nabla f_{i}(x)$ denotes a subgradient of $f(x)$. Thus, the function $g_{i}$ also has a nonempty subdifferential set at any point $(x, \eta)$.

The subgradient error $\varepsilon_{k}^{i}$ in algorithm (10)-(11) is assumed to be stochastic in order to address a general form of the objective function, as in (2), where the subgradient $\nabla f_{i}(x)$ is not readily available to us. We adopt a standard approach in stochastic optimization by using an unbiased estimate $\nabla f_{i}(x)+\bar{\varepsilon}_{k}^{i}$ of the subgradient, where $\bar{\varepsilon}_{k}^{i}$ is a zero mean random variable. Thus, in (11) we have

$$
\varepsilon_{k}^{i}=\bar{\varepsilon}_{k}^{i} \mathbf{1}_{\left\{f_{i}\left(\tilde{x}_{k}^{i}\right) \geq \tilde{\eta}_{k}^{i}\right\}}
$$

The initial points $x_{0}^{i} \in X$ and $\eta_{0}^{i}$, for $i \in V$, may be selected randomly with a distribution independent of any other sources of randomness in the algorithm.

\subsection{Assumptions}

Our assumptions on the network are the same as, for example, those in [29, 32]. 
Assumption 1 For the weight matrices and the communication graphs, we assume the following:

(a) [Weights Rule] There exists a scalar $0<\gamma<1$ such that $\left[W_{k}\right]_{i i} \geq \gamma$ for all $i$ and $k$, and $\left[W_{k}\right]_{i j} \geq \gamma$ whenever $\left[W_{k}\right]_{i j}>0$.

(b)[Doubly Stochasticity] The matrix $W_{k}$ is doubly stochastic for all $k$, i.e., $W_{k} \mathbf{1}=\mathbf{1}$, and $\mathbf{1}^{\prime} W_{k}=\mathbf{1}^{\prime}$.

(c) [Connectedness] There exists an integer $Q \geq 1$ such that the graph with the vertex set $V$ and the edge set $\cup_{\tau=k Q}^{(k+1) Q-1} E(\tau)$ is strongly connected for every $k$.

The assumptions ensure that agent's local variables are properly diffused over timevarying communication networks.

Next, we impose the following assumptions on the subgradients $\nabla f_{i}(x)$ and the errors, where we use $\partial f_{i}(x)$ to denote the set of all subgradients of $f_{i}$ at $x$.

Assumption 2 Let the following hold:

(a) The subgradients of each $f_{i}$ are bounded over the set $X$, i.e., there is a scalar $C>0$ such that $\left\|\nabla f_{i}(x)\right\|_{*} \leq C$ for all $\nabla f_{i}(x) \in \partial f_{i}(x)$, all $x \in X$ and all $i \in V$.

(b) The subgradient errors $\bar{\varepsilon}_{k}^{i}$ when conditioned on the point $x=\tilde{x}_{k}^{i}$ of the subgradient $\nabla f_{i}(x)$ evaluation are zero mean, i.e., $\mathbb{E}\left[\bar{\varepsilon}_{k}^{i} \mid \tilde{x}_{k}^{i}\right]=0$ for all $i \in V$ and $k \geq 0$, with probability 1 .

(c) There is a scalar $v>0$ such that $\mathbb{E}\left[\left\|\bar{\varepsilon}_{k}^{i}\right\|_{*}^{2} \mid \tilde{x}_{k}^{i}\right] \leq v^{2}$ for all $i \in V$ and $k \geq 0$, with probability 1 .

Basically, under Assumptions 2-b and 2-c, the iterations $\left\{x_{k}^{i}\right\}, i \in V$, of the algorithm in (10)-(11) form a Markov process. In what follows, we will use $F_{k}$ to denote the past iterates of the algorithm (11), i.e.,

$$
F_{k}=\left\{x_{t}^{i}, \eta_{t}^{i}, i \in V, t=0,1, \ldots, k\right\} \quad \text { for } k \geq 0 .
$$

Note that, given $F_{k}$, the iterates $\tilde{x}_{k}^{i}$ and $\tilde{\eta}_{k}^{i}$ in (10) are deterministic. In view of this, as a consequence of the subgradient norm and subgradient error boundedness (Assumptions 2-a and 2-c), it can be seen that with probability 1 ,

$$
\mathbb{E}\left[\left\|\nabla f_{i}(x)+\bar{\varepsilon}_{k}^{i}\right\|_{*}^{2} \mid F_{k}\right] \leq(C+v)^{2} \quad \text { for all } i \in V \text { and } k \geq 0 .
$$

Also, as a result of Assumption 2-a, we have

$$
\left\|\nabla_{x} g_{i}\left(\tilde{z}_{k}^{i}\right)\right\|_{*}=\left\|\nabla f_{i}\left(\tilde{x}_{k}^{i}\right)\right\|_{*} \mathbf{1}_{\left\{f_{i}\left(x_{k}^{i}\right) \geq \tilde{\eta}_{k}^{i}\right\}} \leq C \quad \text { for all } i \in V \text { and } k \geq 0 .
$$

This and the zero-mean error assumption (Assumption 2-b) yield

$$
\mathbb{E}\left[\varepsilon_{k}^{i} \mid F_{k}\right]=\mathbb{E}\left[\bar{\varepsilon}_{k}^{i} \mathbf{1}_{\left\{f_{i}\left(\tilde{x}_{k}^{i}\right) \geq \tilde{\eta}_{k}^{i}\right\}} \mid F_{k}\right]=\mathbb{E}\left[\bar{\varepsilon}_{k}^{i} \mid F_{k}\right] \mathbf{1}_{\left\{f_{i}\left(\tilde{x}_{k}^{i}\right) \geq \tilde{\eta}_{k}^{i}\right\}}=0 .
$$

Similarly, as a result of Assumption 2-c we have with probability 1,

$$
\mathbb{E}\left[\left\|\varepsilon_{k}^{i}\right\|_{*}^{2} \mid F_{k}\right]=\mathbb{E}\left[\left\|\bar{\varepsilon}_{k}^{i}\right\|_{*}^{2} \mathbf{1}_{\left\{f_{i}\left(\tilde{x}_{k}^{i}\right) \geq \tilde{\eta}_{k}^{i}\right\}} \mid F_{k}\right]=\mathbb{E}\left[\left\|\bar{\varepsilon}_{k}^{i}\right\|_{*}^{2} \mid F_{k}\right] \mathbf{1}_{\left\{f_{i}\left(\tilde{x}_{k}^{i}\right) \geq \tilde{\eta}_{k}^{i}\right\}} \leq v^{2} .
$$


This, in turn implies that with probability 1 for all $i \in V$ and $k \geq 0$,

$$
\mathbb{E}\left[\left\|\nabla_{x} g_{i}\left(\tilde{z}_{k}^{i}\right)+\varepsilon_{k}^{i}\right\|_{*}^{2} \mid F_{k}\right] \leq(C+v)^{2} .
$$

Applying Jensen's inequality, we find that

$$
\mathbb{E}\left[\left\|\nabla_{x} g_{i}\left(\tilde{z}_{k}^{i}\right)+\varepsilon_{k}^{i}\right\|_{*} \mid F_{k}\right] \leq C+v .
$$

By the definition, a Bregman function is convex in its first variable. We further make the assumption on the choice of Bregman-distance functions that requires convexity with respect to the second variable. We depend on this assumption when showing the convergence of our algorithm.

Assumption 3 Both Bregman-distance functions $B_{x}(y, z)$ and $B_{\eta}(\phi, \zeta)$ are convex in their second arguments $z$ and $\zeta$, respectively, for every fixed $y$ and $\phi$.

\subsection{Algorithm Convergence}

In this section we prove the convergence of algorithm (10)-(11). We use techniques from Lyapunov analysis and the following generalization of the supermartingale convergence theorem, which is also known as the Robbins-Siegmund result as it originated in the work of Robbins and Siegmund [33].

Theorem 2. Let $F_{t}, t=0,1,2, \ldots$, be a filtration such that $F_{t} \subset F_{t+1}$ for $t \geq 0$. Let $\left\{X_{t}\right\},\left\{Y_{t}\right\},\left\{Z_{t}\right\}$ and $\left\{g_{t}\right\}$ be sequences of non-negativerandom variables that are adapted to the filtration $F_{t}$. Assume that for each $t$, we have with probability 1 ,

$$
\mathbb{E}\left[Y_{t+1} \mid F_{t}\right] \leq\left(1+g_{t}\right) Y_{t}-X_{t}+Z_{t},
$$

where $\sum_{t=0}^{\infty} Z_{t}<\infty$ and $\sum_{t=0}^{\infty} g_{t}<\infty$ with probability 1. Then, with probability 1 , $\sum_{t=0}^{\infty} X_{t}<\infty$ and the sequence $Y_{t}$ converges to a nonnegative random variable $Y$.

Our convergence analysis of algorithm (10)-(11) rests on Theorem 2. In order to use this theorem, we establish two main properties of the algorithm showing that the conditions of the theorem are satisfied. We develop these properties in forthcoming Lemmas 4 and 5. In the development, we make use of an alternative representation of the algorithm that relies on transition matrices, defined as follows:

$$
\Phi(k, s)=W_{k} W_{k-1} \cdots W_{s} \quad \text { for all } k, s \text { with } k \geq s \geq 0 .
$$

We next state a result from [22] (Corollary 1) on the convergence properties of the matrix $\Phi(k, s)$.

Lemma 2. Let Assumptions 1 hold. Then, we have $\left|[\Phi(k, s)]_{i j}-\frac{1}{m}\right| \leq \theta \beta^{k-s}$ for all $i, j \in V$ and all $k \geq s \geq 0$, with $\theta=\left(1-\frac{\eta}{4 m^{2}}\right)^{-2}$ and $\beta=\left(1-\frac{\eta}{4 m^{2}}\right)^{\frac{1}{\mathscr{Q}}}$. 
We will also make use of the following result.

Lemma 3. Let $\left\{\gamma_{k}\right\}$ be a non-negative scalar sequence such that $\sum_{k} \gamma_{k}<\infty$. Then, for any $\beta$ with $0<\beta<1$, we have $\sum_{k=0}^{\infty}\left(\sum_{\ell=0}^{k} \beta^{k-\ell} \gamma_{\ell}\right)<\infty$.

Proof. Let $\sum_{k=0}^{\infty} \gamma_{k}<\infty$. For any integer $M \geq 1$, we have $\sum_{k=0}^{M}\left(\sum_{\ell=0}^{k} \beta^{k-\ell} \gamma_{\ell}\right)=$ $\sum_{\ell=0}^{M} \gamma_{\ell} \sum_{t=0}^{M-\ell} \beta^{t} \leq \sum_{\ell=0}^{M} \gamma_{\ell} \frac{1}{1-\beta}$, implying $\sum_{k=0}^{\infty}\left(\sum_{\ell=0}^{k} \beta^{k-\ell} \gamma_{\ell}\right) \leq \frac{1}{1-\beta} \sum_{\ell=0}^{\infty} \gamma_{\ell}<\infty$.

In our analysis, we use auxiliary points, namely the instantaneous averages of the iterates $x_{k}^{i}$ and $\eta_{k}^{i}$ over $i \in V$, defined by

$$
\hat{x}_{k}=\frac{1}{m} \sum_{i=1}^{m} x_{k}^{i}, \quad \hat{\eta}_{k}=\frac{1}{m} \sum_{i=1}^{m} \eta_{k}^{i} \quad \text { for all } k \geq 0 .
$$

We next provide an important result for these averages that we use to assert the convergence properties of our algorithm.

Lemma 4. Let Assumptions 1 and 2 hold, and let $\sum_{k=0}^{\infty} \alpha_{k}^{2}<\infty$. Then, for the iterates of algorithm (10)-(11) we have $\sum_{k=0}^{\infty} \alpha_{k}|| \hat{x}_{k}-x_{k}^{i} \|<\infty$ and $\sum_{k=0}^{\infty} \alpha_{k}\left|\hat{\eta}_{k}-\eta_{k}^{i}\right|<\infty$ for all $i \in V$, with probability 1 .

Proof. Let us denote the noisy subgradient as $\tilde{d}_{k}^{i}=\nabla_{x} g_{i}\left(\tilde{z}_{k}^{i}\right)+\varepsilon_{k}^{i}$. Applying the optimality condition for (11), we get

$$
\left\langle\alpha_{k} r_{i} \tilde{d}_{k}^{i}+\nabla \omega_{x}\left(x_{k+1}^{i}\right)-\nabla \omega_{x}\left(\tilde{x}_{k}^{i}\right), y-x_{k+1}^{i}\right\rangle \geq 0 \quad \text { for all } y \in X .
$$

Since $\tilde{x}_{k}^{i} \in X$, by letting $y=\tilde{x}_{k}^{i}$ we have

$$
\left\langle\alpha_{k} r_{i} \tilde{d}_{k}^{i}+\nabla \omega_{x}\left(x_{k+1}^{i}\right)-\nabla \omega_{x}\left(\tilde{x}_{k}^{i}\right), \tilde{x}_{k}^{i}-x_{k+1}^{i}\right\rangle \geq 0,
$$

which implies

$$
\alpha_{k} r_{i}\left\langle\tilde{d}_{k}^{i}, \tilde{x}_{k}^{i}-x_{k+1}^{i}\right\rangle \geq\left\langle\nabla \omega_{x}\left(\tilde{x}_{k}^{i}\right)-\nabla \omega_{x}\left(x_{k+1}^{i}\right), \tilde{x}_{k}^{i}-x_{k+1}^{i}\right\rangle \geq \sigma_{x}\left\|\tilde{x}_{k}^{i}-x_{k+1}^{i}\right\|^{2},
$$

where the last inequality follows by the strong convexity of $\omega_{x}$. Using Hölder's inequality, we obtain

$$
\alpha_{k} r_{i}\left\|\tilde{d}_{k}^{i}\right\|_{*}\left\|\tilde{x}_{k}^{i}-x_{k+1}^{i}\right\| \geq \sigma_{x}\left\|\tilde{x}_{k}^{i}-x_{k+1}^{i}\right\|^{2} .
$$

Therefore $\left\|\tilde{x}_{k}^{i}-x_{k+1}^{i}\right\| \leq \alpha_{k} r_{i} \frac{\left\|\tilde{d}_{k}^{i}\right\|_{*}}{\sigma_{x}}$, and taking the conditional expectation yields

$$
\mathbb{E}\left[\left\|\tilde{x}_{k}^{i}-x_{k+1}^{i}\right\| \mid F_{k}\right] \leq \alpha_{k} r_{i} \frac{\mathbb{E}\left[\left\|\tilde{d}_{k}^{i}\right\|_{*} \mid F_{k}\right]}{\sigma_{x}} \leq \alpha_{k} r_{i} \frac{C+v}{\sigma_{x}},
$$

where the last inequality follows from (14) under Assumptions 2-a and 2-c.

Let us now write the iterates as follows 
Distributed Bregman-Distance Algorithms for Min-Max Optimization

$$
x_{k+1}^{i}=\tilde{x}_{k}^{i}+e_{k}^{i} \quad \text { with } \quad e_{k}^{i}=x_{k+1}^{i}-\tilde{x}_{k}^{i} .
$$

By (16) and the iterated expectation rule, for $e_{k}^{i}$ we obtain

$$
\mathbb{E}\left[\left\|e_{k}^{i}\right\|\right]=\mathbb{E}\left[\mathbb{E}\left[\left\|e_{k}^{i}\right\| \mid F_{k}\right]\right] \leq \alpha_{k} r_{i} \frac{C+v}{\sigma_{x}} \quad \text { for all } i \text { and } k .
$$

By taking the average of the relations in (17) over $i=1, \ldots, m$ and using the doubly stochastic property of $W_{k}$, we can see that for all $k$,

$$
\hat{x}_{k+1}=\hat{x}_{k}+\frac{1}{m} \sum_{i=1}^{m} e_{k}^{i}
$$

Now, we note that by (17) and the definition of $\tilde{x}_{k}^{i}$, we have $x_{k+1}^{i}=\sum_{j=1}^{m}\left[W_{k}\right]_{i j} x_{k}^{j}+e_{k}^{i}$. We then recursively use this equation to relate $x_{k+1}^{i}$ and $x_{0}^{j}$ for $j \in V$. We do so by using the matrices $\Phi(k, s)$ defined in (15) to obtain

$$
x_{k+1}^{i}=\sum_{j=1}^{m}[\Phi(k, 0)]_{i j} x_{0}^{j}+e_{k}^{i}+\sum_{\ell=0}^{k-1}\left(\sum_{j=1}^{m}[\Phi(k, \ell+1)]_{i j} e_{\ell}^{j}\right) .
$$

Similarly, we derive the recursive relation for $\hat{x}_{k+1}$ as given in (19), and obtain:

$$
\hat{x}_{k+1}=\hat{x}_{0}+\frac{1}{m} \sum_{\ell=0}^{k} \sum_{j=1}^{m} e_{\ell}^{j}=\frac{1}{m} \sum_{j=1}^{m} x_{0}^{j}+\frac{1}{m} \sum_{j=1}^{m} e_{k}^{j}+\frac{1}{m} \sum_{\ell=0}^{k-1} \sum_{j=1}^{m} e_{\ell}^{j} .
$$

Then, from (20) and (21) we have

$$
\begin{aligned}
\left\|x_{k+1}^{i}-\hat{x}_{k+1}\right\| \leq & \left\|\sum_{j=1}^{m}\left([\Phi(k, 0)]_{i j}-\frac{1}{m}\right) x_{0}^{j}\right\|+\left\|e_{k}^{i}-\frac{1}{m} \sum_{j=1}^{m} e_{k}^{j}\right\| \\
& +\left\|\sum_{\ell=0}^{k-1} \sum_{j=1}^{m}\left([\Phi(k, \ell+1)]_{i j}-\frac{1}{m}\right) e_{\ell}^{j}\right\| .
\end{aligned}
$$

Therefore, for all $j \in V$ and all $k$,

$$
\begin{aligned}
\left\|x_{k+1}^{i}-\hat{x}_{k+1}\right\| \leq & \sum_{j=1}^{m}\left|[\Phi(k, 0)]_{i j}-\frac{1}{m}\right|\left\|x_{0}^{j}\right\|+\frac{1}{m} \sum_{j \neq i}\left\|e_{k}^{i}-e_{k}^{j}\right\| \\
& +\sum_{\ell=0}^{k-1} \sum_{j=1}^{m}\left|[\Phi(k, \ell+1)]_{i j}-\frac{1}{m}\right|\left\|e_{\ell}^{j}\right\| .
\end{aligned}
$$

At this point, we use the rate of convergence result from Lemma 2 to bound $\left|[\Phi(k, \ell)]_{i j}-\frac{1}{m}\right|$. By doing so, we obtain

$$
\left\|x_{k+1}^{i}-\hat{x}_{k+1}\right\| \leq \theta \beta^{k} \sum_{j=1}^{m}\left\|x_{0}^{j}\right\|+\frac{1}{m} \sum_{j \neq i}\left\|e_{k}^{i}-e_{k}^{j}\right\|+\theta \sum_{\ell=0}^{k-1} \sum_{j=1}^{m} \beta^{k-(\ell+1)}\left\|e_{\ell}^{j}\right\|
$$


where $\beta<1$ (see Lemma 2). By taking the expectation and using (18) we find that for all $i$ and $k$,

$$
\mathbb{E}\left[\left\|x_{k+1}^{i}-\hat{x}_{k+1}\right\|\right] \leq \theta \beta^{k} \sum_{j=1}^{m} \mathbb{E}\left[\left\|x_{0}^{j}\right\|\right]+\frac{2(m-1) c}{m} \alpha_{k}+m \theta c \sum_{\ell=0}^{k-1} \beta^{k-(\ell+1)} \alpha_{\ell},
$$

with $c=\left(\max _{i \in V} r_{i}\right)(C+v) / \sigma_{x}$. Next, we multiply the preceding relation with $\alpha_{k+1}$ and after using $a b \leq\left(a^{2}+b^{2}\right) / 2$ for the terms $\alpha_{k+1} \alpha_{k}$ and $\alpha_{k+1} \alpha_{\ell}$, we obtain

$$
\begin{aligned}
\alpha_{k+1} \mathbb{E}\left[\left\|x_{k+1}^{i}-\hat{x}_{k+1}\right\|\right] \leq & \theta \alpha_{k+1} \beta^{k} \sum_{j=1}^{m} \mathbb{E}\left[\left\|x_{0}^{j}\right\|\right]+\frac{(m-1) c}{m}\left(\alpha_{k}^{2}+\alpha_{k+1}^{2}\right) \\
& +m \frac{\theta c}{2} \sum_{\ell=0}^{k-1} \beta^{k-(\ell+1)}\left(\alpha_{k+1}^{2}+\alpha_{\ell}^{2}\right)
\end{aligned}
$$

We observe that by $\sum_{k=0}^{\infty} \alpha_{k}^{2}<\infty$ it follows that $\alpha_{k} \rightarrow 0$, and hence $\alpha_{k}$ is bounded. This and the fact $\beta<1$ imply $\sum_{k=0}^{\infty} \alpha_{k+1} \beta^{k}<\infty$. The sum $\sum_{k=0}^{\infty}\left(\alpha_{k}^{2}+\alpha_{k+1}^{2}\right)$ is obviously summable when $\sum_{k=0}^{\infty} \alpha_{k}^{2}<\infty$. For the last term, by $\beta<1$ we have

$$
\sum_{k=1}^{\infty} \sum_{\ell=0}^{k-1} \beta^{k-(\ell+1)}\left(\alpha_{k+1}^{2}+\alpha_{\ell}^{2}\right) \leq \sum_{k=1}^{\infty} \frac{\alpha_{k+1}^{2}}{1-\beta}+\sum_{k=1}^{\infty} \sum_{\ell=0}^{k-1} \beta^{k-(\ell+1)} \alpha_{\ell}^{2} .
$$

The first sum is finite since $\sum_{k=0}^{\infty} \alpha_{k}^{2}<\infty$, while the second sum is finite by Lemma 3 . Thus, $\sum_{k=0}^{\infty} \alpha_{k+1} \mathbb{E}\left[\left\|x_{k+1}^{i}-\hat{x}_{k+1}\right\|\right]<\infty$ for all $i \in V$ and by the monotone convergence theorem [7], it follows $\mathbb{E}\left[\sum_{k=0}^{\infty} \alpha_{k+1}\left\|x_{k+1}^{i}-\hat{x}_{k+1}\right\|\right]<\infty$. When the expected value of a positive random variable is finite, then the variable must be finite with probability 1 , so we must have $\sum_{k=0}^{\infty} \alpha_{k+1}\left\|x_{k+1}^{i}-\hat{x}_{k+1}\right\|<\infty$ for all $i \in V$, with probability 1 .

A similar analysis proves $\sum_{k=0}^{\infty} \alpha_{k}\left|\hat{\eta}_{k}-\eta_{k}^{i}\right|<\infty$ for all $i \in V$, with probability 1 .

Lemma 4 provides one important property of the iterates generated by our algorithm. We next provide another important relation that in a way captures a descent property of the iterates in terms of the Lyapunov function given by the sum of the Bregman-distance functions $B_{x}$ and $B_{\eta}$. For notational convenience, we define

$$
z_{k}^{i}=\left[\begin{array}{l}
x_{k}^{i} \\
\eta_{k}^{i}
\end{array}\right], \quad \hat{z}_{k}=\left[\begin{array}{l}
\hat{x}_{k} \\
\hat{\eta}_{k}
\end{array}\right] \quad \text { for all } k \geq 0 .
$$

Also, we introduce the notation $\mathbb{E}_{k}[\cdot]=\mathbb{E}\left[\cdot \mid F_{k}\right]$.

We have the following relation for algorithm (10)-(11), under the assumption that the Bregman-distance functions $B_{x}$ and $B_{\eta}$ are convex in their second arguments.

Lemma 5. Let Assumptions 1, 2 and 3 hold. Then, for algorithm (10)-(11) we have with probability 1 for any $x^{*} \in X^{*}$ and all $k \geq 0$, 


$$
\begin{aligned}
& \sum_{i=1}^{m} \mathbb{E}_{k}\left[B_{x}\left(x^{*}, x_{k+1}^{i}\right)+B_{\eta}\left(\eta^{*}, \eta_{k+1}^{i}\right)\right] \leq \sum_{i=1}^{m}\left(B_{x}\left(x^{*}, x_{k}^{i}\right)+B_{\eta}\left(\eta^{*}, \eta_{k}^{i}\right)\right) \\
& -\alpha_{k}\left(\sum_{i=1}^{m} r_{i} g_{i}\left(\hat{z}_{k}\right)+\hat{\eta}_{k}-\eta^{*}\right)+\alpha_{k} \bar{r}\left(C \sum_{j=1}^{m}\left\|\hat{x}_{k}-x_{k}^{j}\right\|+\sum_{j=1}^{m}\left|\hat{\eta}_{k}-\eta_{k}^{j}\right|\right) \\
& +\alpha_{k}^{2} m\left(\frac{\bar{r}^{2}(C+v)^{2}}{2 \sigma_{x}}+\frac{\left(\frac{1}{m}+\bar{r}\right)^{2}}{2 \sigma_{\eta}}\right)
\end{aligned}
$$

where $\bar{r}=\max _{i \in V} r_{i}$.

Proof. By the definition of the Bregman function $B_{x}$, we have

$$
\begin{gathered}
B_{x}\left(x^{*}, x_{k+1}^{i}\right)-B_{x}\left(x^{*}, \tilde{x}_{k}^{i}\right)=w_{x}\left(\tilde{x}_{k}^{i}\right)-w_{x}\left(x_{k+1}^{i}\right)-\left\langle\nabla w_{x}\left(x_{k+1}^{i}\right), x^{*}-x_{k+1}^{i}\right\rangle \\
+\left\langle\nabla w_{x}\left(\tilde{x}_{k}^{i}\right), x^{*}-\tilde{x}_{k}^{i}\right\rangle .
\end{gathered}
$$

Noting that $w_{x}\left(\tilde{x}_{k}^{i}\right)-w_{x}\left(x_{k+1}^{i}\right)=\left\langle\nabla w_{x}\left(\tilde{x}_{k}^{i}\right), \tilde{x}_{k}^{i}-x_{k+1}^{i}\right\rangle-B_{x}\left(x_{k+1}^{i}, \tilde{x}_{k}^{i}\right)$, we obtain

$$
B_{x}\left(x^{*}, x_{k+1}^{i}\right)-B_{x}\left(x^{*}, \tilde{x}_{k}^{i}\right)=\left\langle\nabla w_{x}\left(\tilde{x}_{k}^{i}\right)-\nabla w_{x}\left(x_{k+1}^{i}\right), x^{*}-x_{k+1}^{i}\right\rangle-B_{x}\left(x_{k+1}^{i}, \tilde{x}_{k}^{i}\right) .
$$

Now, using the optimality condition for (11), since $x^{*} \in X$, we have $\left\langle\alpha_{k} r_{i} \tilde{d}_{k}^{i}+\right.$ $\left.\nabla \omega_{x}\left(x_{k+1}^{i}\right)-\nabla \omega_{x}\left(\tilde{x}_{k}^{i}\right), x^{*}-x_{k+1}^{i}\right\rangle \geq 0$, where $\tilde{d}_{k}^{i}=\nabla_{x} g_{i}\left(\tilde{z}_{k}^{i}\right)+\varepsilon_{k}^{i}$. This implies

$$
\left\langle\nabla \omega_{x}\left(\tilde{x}_{k}^{i}\right)-\nabla \omega_{x}\left(x_{k+1}^{i}\right), x^{*}-x_{k+1}^{i}\right\rangle \leq \alpha_{k} r_{i}\left\langle\tilde{d}_{k}^{i}, x^{*}-x_{k+1}^{i}\right\rangle .
$$

Upon substituting the preceding inequality in (23), we obtain

$$
\begin{aligned}
B_{x}\left(x^{*}, x_{k+1}^{i}\right) & -B_{x}\left(x^{*}, \tilde{x}_{k}^{i}\right) \leq \alpha_{k} r_{i}\left\langle\tilde{d}_{k}^{i}, x^{*}-x_{k+1}^{i}\right\rangle-B_{x}\left(x_{k+1}^{i}, \tilde{x}_{k}^{i}\right) \\
& \leq \alpha_{k} r_{i}\left\langle\tilde{d}_{k}^{i}, x^{*}-\tilde{x}_{k}^{i}\right\rangle+\alpha_{k} r_{i}\left\langle\tilde{d}_{k}^{i}, \tilde{x}_{k}^{i}-x_{k+1}^{i}\right\rangle-\frac{\sigma_{x}}{2}\left\|x_{k+1}^{i}-\tilde{x}_{k}^{i}\right\|^{2} .
\end{aligned}
$$

where the last inequality follows from the strong convexity of the Bregman function, i.e., $B_{x}\left(x_{k+1}^{i}, \tilde{x}_{k}^{i}\right) \geq \frac{\sigma_{x}}{2}\left\|x_{k+1}^{i}-\tilde{x}_{k}^{i}\right\|^{2}$. Further, by Hölder's inequality we have $\alpha_{k} r_{i}\left\langle\tilde{d}_{k}^{i}, \tilde{x}_{k}^{i}-x_{k+1}^{i}\right\rangle \leq \alpha_{k} r_{i}\left\|\tilde{d}_{k}^{i}\right\|_{*}\left\|\tilde{x}_{k}^{i}-x_{k+1}^{i}\right\|$. Using this and the scalar inequality $2 a b \leq a^{2}+b^{2}$, we obtain

$$
\begin{aligned}
\alpha_{k} r_{i}\left\langle\tilde{d}_{k}^{i}, \tilde{x}_{k}^{i}-x_{k+1}^{i}\right\rangle & \leq 2 \frac{\alpha_{k} r_{i}}{\sqrt{2 \sigma_{x}}}\left\|\tilde{d}_{k}^{i}\right\|_{*} \frac{\sqrt{\sigma_{x}}}{\sqrt{2}}\left\|\tilde{x}_{k}^{i}-x_{k+1}^{i}\right\| \\
& \leq \alpha_{k}^{2} r_{i}^{2} \frac{\left\|\tilde{d}_{k}^{i}\right\|_{*}^{2}}{2 \sigma_{x}}+\frac{\sigma_{x}}{2}\left\|x_{k+1}^{i}-\tilde{x}_{k}^{i}\right\|^{2} .
\end{aligned}
$$

Upon combining (25) and (24) we arrive at

$$
B_{x}\left(x^{*}, x_{k+1}^{i}\right)-B_{x}\left(x^{*}, \tilde{x}_{k}^{i}\right) \leq \alpha_{k} r_{i}\left\langle\tilde{d}_{k}^{i}, x^{*}-\tilde{x}_{k}^{i}\right\rangle+\alpha_{k}^{2} r_{i}^{2} \frac{\left\|\tilde{d}_{k}^{i}\right\|_{*}^{2}}{2 \sigma_{x}} .
$$


Since $\tilde{d}_{k}^{i}=\nabla_{x} g_{i}\left(\tilde{z}_{k}^{i}\right)+\varepsilon_{k}^{i}$, by taking the conditional expectation with respect to the history $F_{k}$, and by using (13) and $\mathbb{E}_{k}\left[\tilde{d}_{k}^{i}\right]=\nabla_{x} g_{i}\left(v_{k}^{i}\right)$, we obtain

$$
\mathbb{E}_{k}\left[B_{x}\left(x^{*}, x_{k+1}^{i}\right)\right] \leq B_{x}\left(x^{*}, \tilde{x}_{k}^{i}\right)-\alpha_{k} r_{i}\left\langle\nabla_{x} g_{i}\left(\tilde{z}_{k}^{i}\right), \tilde{x}_{k}^{i}-x^{*}\right\rangle+\alpha_{k}^{2} r_{i}^{2} \frac{(C+v)^{2}}{2 \sigma_{x}} .
$$

Proceeding similarly, we can derive the following inequality for the iterates involving the min-max value estimates $\eta_{k}^{i}$,

$\mathbb{E}_{k}\left[B_{\eta}\left(\eta^{*}, \eta_{k+1}^{i}\right)\right] \leq B_{\eta}\left(\eta^{*}, \tilde{\eta}_{k}^{i}\right)-\alpha_{k}\left(\frac{1}{m}+r_{i} \nabla_{\eta} g_{i}\left(\tilde{z}_{k}^{i}\right)\right)\left(\tilde{\eta}_{k}^{i}-\eta^{*}\right)+\alpha_{k}^{2} \frac{\left(\frac{1}{m}+r_{i}\right)^{2}}{2 \sigma_{\eta}}$,

where we use the fact $\left|\nabla_{\eta} g_{i}\left(\tilde{z}_{k}^{i}\right)\right| \leq 1$. By the convexity of $g_{i}$ and the subgradient property, we have

$$
\nabla g_{i}\left(\tilde{z}_{k}^{i}\right)^{\prime}\left(\tilde{z}_{k}^{i}-z^{*}\right) \geq g_{i}\left(\tilde{z}_{k}^{i}\right)-g_{i}\left(z^{*}\right)=g_{i}\left(\tilde{z}_{k}^{i}\right) .
$$

where the equality follows by $g_{i}\left(z^{*}\right)=\max \left\{0, f_{i}\left(x^{*}\right)-\eta^{*}\right\}$ and $f_{i}\left(x^{*}\right)-\eta^{*} \leq 0$ for all $i \in V$ and any optimal point $x^{*} \in X^{*}$. Upon adding the inequalities (26) and (27), and using the convexity of $g_{i}$, we obtain for any $x^{*} \in X^{*}$ and all $k \geq 0$ and $i \in V$,

$$
\begin{gathered}
\mathbb{E}_{k}\left[B_{x}\left(x^{*}, x_{k+1}^{i}\right)+B_{\eta}\left(\eta^{*}, \eta_{k+1}^{i}\right)\right] \leq B_{x}\left(x^{*}, \tilde{x}_{k}^{i}\right)+B_{\eta}\left(\eta^{*}, \tilde{\eta}_{k}^{i}\right)-\alpha_{k} \frac{1}{m}\left(\tilde{\eta}_{k}^{i}-\eta^{*}\right) \\
-\alpha_{k} r_{i} g_{i}\left(\tilde{z}_{k}^{i}\right)+\alpha_{k}^{2}\left(\frac{r_{i}^{2}(C+v)^{2}}{2 \sigma_{x}}+\frac{\left(\frac{1}{m}+r_{i}\right)^{2}}{2 \sigma_{\eta}}\right) .
\end{gathered}
$$

Now, by Assumption 3 on the convexity of the Bregman functions $B_{x}$ and $B_{\eta}$ and the doubly stochasticity of the weight matrix $W_{k}$ (Assumption 1-b), we have

$$
\sum_{i=1}^{m} B_{x}\left(x^{*}, \tilde{x}_{k}^{i}\right)=\sum_{i=1}^{m} B_{x}\left(x^{*}, \sum_{j=1}^{m}\left[W_{k}\right]_{i j} x_{k}^{j}\right) \leq \sum_{i=1}^{m} \sum_{j=1}^{m}\left[W_{k}\right]_{i j} B_{x}\left(x^{*}, x_{k}^{j}\right)=\sum_{j=1}^{m} B_{x}\left(x^{*}, x_{k}^{j}\right) .
$$

Similarly, we have $\sum_{i=1}^{m} B_{\eta}\left(\eta^{*}, \tilde{\eta}_{k}^{i}\right) \leq \sum_{j=1}^{m} B_{\eta}\left(\eta^{*}, \eta_{k}^{j}\right)$. Thus, by summing inequalities in (28) over $i \in V$ we obtain for any $x^{*} \in X^{*}$ and all $k \geq 0$,

$$
\begin{aligned}
& \sum_{i=1}^{m} \mathbb{E}_{k}\left[B_{x}\left(x^{*}, x_{k+1}^{i}\right)+B_{\eta}\left(\eta^{*}, \eta_{k+1}^{i}\right)\right] \leq \sum_{i=1}^{m}\left(B_{x}\left(x^{*}, x_{k}^{i}\right)+B_{\eta}\left(\eta^{*}, \eta_{k}^{i}\right)\right) \\
& -\alpha_{k} \frac{1}{m} \sum_{i=1}^{m}\left(\tilde{\eta}_{k}^{i}-\eta^{*}\right)-\alpha_{k} \sum_{i=1}^{m} r_{i} g_{i}\left(\tilde{z}_{k}^{i}\right)+\alpha_{k}^{2} m\left(\frac{\bar{r}^{2}(C+v)^{2}}{2 \sigma_{x}}+\frac{\left(\frac{1}{m}+\bar{r}\right)^{2}}{2 \sigma_{\eta}}\right),
\end{aligned}
$$

where $\bar{r}=\max _{i \in V} r_{i}$.

From the definition of $\tilde{\eta}_{k}^{i}$ and the doubly stochasticity of the matrix $W_{k}$, we have $\sum_{i=1}^{m} \tilde{\eta}_{k}^{i}=\sum_{i=1}^{m} \sum_{j=1}^{m}\left[W_{k}\right]_{i j} \eta_{k}^{j}=\sum_{j=1}^{m} \eta_{k}^{j}=m \hat{\eta}_{k}$. Using this identity, together with 
adding and subtracting the term $\alpha_{k} \sum_{i=1}^{m} r_{i} g_{i}\left(\hat{z}_{k}\right)$, from the preceding relation we obtain

$$
\begin{aligned}
& \sum_{i=1}^{m} \mathbb{E}_{k}\left[B_{x}\left(x^{*}, x_{k+1}^{i}\right)+B_{\eta}\left(\eta^{*}, \eta_{k+1}^{i}\right)\right] \leq \sum_{i=1}^{m}\left(B_{x}\left(x^{*}, x_{k}^{i}\right)+B_{\eta}\left(\eta^{*}, \eta_{k}^{i}\right)\right) \\
& -\alpha_{k}\left(\sum_{i=1}^{m} r_{i} g_{i}\left(\hat{z}_{k}\right)+\hat{\eta}_{k}-\eta^{*}\right)+\alpha_{k} \sum_{i=1}^{m} r_{i}\left|g_{i}\left(\hat{z}_{k}\right)-g_{i}\left(\tilde{z}_{k}^{i}\right)\right| \\
& +\alpha_{k}^{2} m\left(\frac{\bar{r}^{2}(C+v)^{2}}{2 \sigma_{x}}+\frac{\left(\frac{1}{m}+\bar{r}\right)^{2}}{2 \sigma_{\eta}}\right)
\end{aligned}
$$

where $\hat{z}_{k}$ is as defined in (22). Next, we consider the term $\sum_{i=1}^{m} r_{i}\left|g_{i}\left(\hat{z}_{k}\right)-g_{i}\left(\tilde{z}_{k}^{i}\right)\right|$. By the definition of $g_{i}$ and relation $|\max \{a, 0\}-\max \{b, 0\}| \leq|a-b|$ valid for any two scalars $a$ and $b$, we have the following:

$$
\left|g_{i}\left(\hat{z}_{k}\right)-g_{i}\left(\tilde{z}_{k}^{i}\right)\right| \leq\left|f_{i}\left(\hat{x}_{k}\right)-f_{i}\left(\tilde{x}_{k}^{i}\right)-\hat{\eta}_{k}+\tilde{\eta}_{k}^{i}\right| \leq C|| \hat{x}_{k}-\tilde{x}_{k}^{i} \|+\left|\hat{\eta}_{k}-\tilde{\eta}_{k}^{i}\right|,
$$

where in the first inequality we use the definition of $\tilde{z}_{k}^{i}$ in (10), while in the last inequality we use the subgradient boundedness assumption for $f_{i}$. Further, by using the definition of the variables $\tilde{x}_{k}^{i}$ and $\tilde{\eta}_{k}^{i}$ in (10), the stochasticity of $W_{k}$ and the convexity of the norm, we obtain

$$
\left|g_{i}\left(\hat{z}_{k}\right)-g_{i}\left(\tilde{z}_{k}^{i}\right)\right| \leq \sum_{j=1}^{m}\left[W_{k}\right]_{i j}\left[C\left\|\hat{x}_{k}-x_{k}^{j}\right\|+\left|\hat{\eta}_{k}-\eta_{k}^{j}\right|\right] .
$$

Therefore, by using the doubly stochasticity of $W_{k}$ and $\bar{r}=\max r_{i}$, we obtain

$$
\sum_{i=1}^{m} r_{i}\left|g_{i}\left(\hat{z}_{k}\right)-g_{i}\left(\tilde{z}_{k}^{i}\right)\right| \leq \bar{r} C \sum_{j=1}^{m}\left\|\hat{x}_{k}-x_{k}^{j}\right\|+\bar{r} \sum_{j=1}^{m}\left|\hat{\eta}_{k}-\eta_{k}^{j}\right| .
$$

Substituting this estimate back in (30) we get the desired result.

We are now ready to prove our main convergence result. The result essentially states that under suitable conditions on the step size $\alpha_{k}$, all the agents' estimates converge to a common optimal point. Moreover, the agents' estimates of the minmax value also converge to the optimal value of the problem.

Theorem 3. Let Assumptions 1, 2 , and 3 hold. Let the step sizes satisfy $\sum_{k=0}^{\infty} \alpha_{k}=\infty$ and $\sum_{k=0}^{\infty} \alpha_{k}^{2}<\infty$. Then, for all $i \in V$, the agents' iterates $x_{k}^{i}$ and $\eta_{k}^{i}$ generated by algorithm (10)-(11) are such that with probability 1 for all $i \in V$ :

(a) The decision variables $x_{k}^{i}$ converge to a common optimal (random) point $x^{*} \in X^{*}$.

(b) The estimates $\eta_{k}^{i}$ converge to the optimal value $\eta^{*}$ of the min-max problem.

Proof. Our analysis is based on applying the Robbins-Siegmund result from Theorem 2 to the inequality derived in Lemma 5 . By our assumption on the step sizes we have $\sum_{k=0}^{\infty} \alpha_{k}^{2}<\infty$, which trivially implies that 


$$
\sum_{k=0}^{\infty} \alpha_{k}^{2} m\left(\frac{\bar{r}^{2}(C+v)^{2}}{2 \sigma_{x}}+\frac{\left(\frac{1}{m}+\bar{r}\right)^{2}}{2 \sigma_{\eta}}\right)<\infty .
$$

Furthermore, by virtue of Lemma 4 we have

$$
\sum_{k=0}^{\infty} \alpha_{k} \bar{r} \sum_{j=1}^{m}\left(C\left\|\hat{x}_{k}-x_{k}^{j}\right\|+\left|\hat{\eta}_{k}-\eta_{k}^{j}\right|\right)<\infty
$$

In addition, by the definition $\hat{z}_{k}^{i}$ is a convex combination of $\left(x_{k}^{i}, \eta_{k}^{i}\right) \in X \times \mathbb{R}$ for all $i \in V$ and $k$, implying by Lemma 1 that $\sum_{i=1}^{m} r_{i} g_{i}\left(\hat{z}_{k}\right)+\hat{\eta}_{k}-\eta^{*} \geq 0$ for all $k \geq$ 0 . Thus, we can apply Lemma 2 to the relation of Lemma 5 and infer that with probability $1, \sum_{i=1}^{m}\left(B_{x}\left(x^{*}, x_{k}^{i}\right)+B_{\eta}\left(\eta^{*}, \eta_{k}^{i}\right)\right)$ converges for every $x^{*} \in X^{*}$ and

$$
\sum_{k=0}^{\infty} \alpha_{k}\left(\sum_{i=1}^{m} r_{i} g_{i}\left(\hat{z}_{k}\right)+\hat{\eta}_{k}-\eta^{*}\right)<\infty .
$$

Now, since $\sum_{k=0}^{\infty} \alpha_{k}=\infty$, from (31)-(32) it follows that there exists a subsequence indexed by $\left\{k_{\ell}\right\}$ such that with probability 1 ,

$$
\begin{aligned}
& \lim _{\ell \rightarrow \infty}\left(\sum_{i=1}^{m} r_{i} g_{i}\left(\hat{z}_{k_{\ell}}\right)+\hat{\eta}_{k_{\ell}}-\eta^{*}\right)=0, \\
& \lim _{\ell \rightarrow \infty}\left\|\hat{x}_{k_{\ell}}-x_{k_{\ell}}^{j}\right\|=0 \text { and } \lim _{\ell \rightarrow \infty}\left|\hat{\eta}_{k_{\ell}}-\eta_{k_{\ell}}^{j}\right|=0 \text { for all } j .
\end{aligned}
$$

Since $\sum_{i=1}^{m}\left(B_{x}\left(x^{*}, x_{k}^{i}\right)+B_{\eta}\left(\eta^{*}, \eta_{k}^{i}\right)\right)$ converges for every $x^{*} \in X^{*}$, the sequence $\sum_{i=1}^{m}\left(B_{x}\left(x^{*}, x_{k}^{i}\right)+B_{\eta}\left(\eta^{*}, \eta_{k}^{i}\right)\right)$ must be bounded for every $x^{*} \in X^{*}$ with probability 1 . Note that from Assumption 3 on the convexity of Bregman functions $B_{x}$ and $B_{\eta}$ and their inherent strong convexity property we have

$$
\begin{aligned}
\frac{1}{m} \sum_{i=1}^{m}\left(B_{x}\left(x^{*}, x_{k}^{i}\right)+B_{\eta}\left(\eta^{*}, \eta_{k}^{i}\right)\right) & \geq B_{x}\left(x^{*}, \hat{x}_{k}\right)+B_{\eta}\left(\eta^{*}, \hat{\eta}_{k}\right) \\
& \geq \frac{\sigma_{x}}{2}\left\|x^{*}-\hat{x}_{k}\right\|^{2}+\frac{\sigma_{\eta}}{2}\left|\eta^{*}-\hat{\eta}_{k}\right|^{2}
\end{aligned}
$$

Thus, the sequences $\left\{\hat{x}_{k}\right\}$ and $\left\{\hat{\eta}_{k}\right\}$ are also bounded with probability 1 . Hence, along a further subsequence, which without loss of generality we can let it be indexed by the same index set $\left\{k_{\ell}, \ell=1,2, \ldots\right\}$, with probability 1 we have $\lim _{\ell \rightarrow \infty} \hat{x}_{k_{\ell}}=$ $\bar{x}$ and $\lim _{\ell \rightarrow \infty} \hat{\eta}_{k \ell}=\bar{\eta}$, where $\bar{x} \in X$ since $X$ is closed. Moreover, with probability 1 the limit points satisfy

$$
\sum_{i=1}^{m} r_{i} g_{i}(\bar{x})+\bar{\eta}-\eta^{*}=0
$$

From this relation and Lemma 1 it follows that $\bar{x} \in X^{*}$ and $\bar{\eta}=\eta^{*}$ with probability 1 . 
In view of (33), and $\hat{x}_{k_{\ell}} \rightarrow \bar{x}$ and $\hat{\eta}_{k_{\ell}} \rightarrow \eta^{*}$, we further have $x_{k_{\ell}}^{j} \rightarrow \bar{x}$ and $\eta_{k_{\ell}}^{j} \rightarrow \bar{\eta}$ for all $j$ with probability 1 . Therefore, $\lim _{\ell \rightarrow \infty} \sum_{i=1}^{m}\left(B_{x}\left(\bar{x}, x_{k_{\ell}}^{i}\right)+B_{\eta}\left(\eta^{*}, \eta_{k_{\ell}}^{i}\right)\right)=$ 0 . However, we have established that the sequence $\sum_{i=1}^{m}\left(B_{x}\left(x^{*}, x_{k}^{i}\right)+B_{\eta}\left(\eta^{*}, \eta_{k}^{i}\right)\right)$ converges with probability 1 for any $x^{*} \in X^{*}$, which in view of $\bar{x} \in X^{*}$, implies that with probability 1 ,

$$
\lim _{k \rightarrow \infty} \sum_{i=1}^{m}\left(B_{x}\left(\bar{x}, x_{k}^{i}\right)+B_{\eta}\left(\eta^{*}, \eta_{k}^{i}\right)\right)=0 .
$$

Finally, by the strong convexity of the Bregman-distance functions $B_{x}$ and $B_{\eta}$, it follows that $x_{k}^{j} \rightarrow \bar{x}$ and $\eta_{k}^{j} \rightarrow \eta^{*}$ with probability 1 for all $j$.

One may extend algorithm (10)-(11) to the case when each agent $i$ uses a different Bregman function $B_{\eta, i}$ instead of a common one $B_{\eta}$. Following the same analysis, with a slight modification, it can be seen that the convergence result of Theorem 3 would be applicable to such a modified algorithm.

\section{Primal-Dual Approach}

In this section we present a distributed primal-dual algorithm which is motivated by the classical work of Arrow-Hurwicz-Uzawa [2]. Recently, a primal-dual method was studied in [24] for approximate solutions to saddle-point problems by considering standard Euclidean norm. The use of Bregman distance for a saddle-point problem was studied in [26]. The prior work is dealing with centralized problems, while here we consider a primal-dual method with Bregman distances for solving the distributed min-max problem in its epigraph formulation (3).

In order to apply primal-dual approach, we need to slightly modify the original epigraph formulation (3) of the min-max problem but without changing its set of optimal solutions. This is needed to ensure the convergence of the primal-dual algorithm. Specifically, we assume that we have a closed convex interval $D$ such that $\eta^{*} \in D$, which is equivalent to having some (arbitrarily large) upper and lower estimates for $\eta^{*}$. Having such a set, we consider a modification of epigraph formulation (3), given by

$$
\begin{array}{ll}
\operatorname{minimize} & \eta \\
\text { subject to } & f_{i}(x) \leq \eta \quad \text { for all } x \in X, \eta \in D, \text { and } i \in V .
\end{array}
$$

This problem has the same solutions as the original min-max problem since $\eta^{*} \in D$. The Lagrangian function associated with this problem is given by

$$
\mathscr{L}(x, \eta, \mu)=\sum_{i=1}^{m} \mu_{i}\left(f_{i}(x)-\eta\right)+\eta \quad \text { for } x \in X, \eta \in D, \mu \geq 0 .
$$


We can restrict the domain of dual variables of the Lagrangian function to the unit interval, as we know that the dual optimal multipliers satisfy $\sum_{i=1}^{m} \mu_{i}^{*}=1$, as seen in Section 3. Thus, we will consider the Lagrangian function with a restricted domain (yet large enough to contain all dual optimal solutions):

$$
\mathscr{L}(x, \eta, \mu)=\sum_{i=1}^{m} \mu_{i}\left(f_{i}(x)-\eta\right)+\eta \quad \text { for } x \in X, \eta \in D, \mu \in I^{m},
$$

where $I$ is the interval $[0,1]$ and $I^{m}$ denotes the product of $m$ copies of $I$. We are interested in determining a primal-dual optimal pair for problem (34) by an algorithm aimed at finding a saddle-point of the reduced Lagrangian (35).

\subsection{Primal-Dual Algorithm}

We are interested in distributed algorithm for computing a saddle-point of the Lagrangian (35). To accommodate distributed computations among $m$ agents, we write the Lagrangian function as a sum of $m$ functions, as follows:

$$
L(x, \eta, \mu)=\sum_{i=1}^{m} \mu_{i}\left(f_{i}(x)-\eta\right)+\eta=\sum_{i=1}^{m}\left(\mu_{i}\left(f_{i}(x)-\eta\right)+\frac{1}{m} \eta\right) .
$$

Lagrangian-function component $L_{i}\left(x, \eta, \mu_{i}\right)=\mu_{i}\left(f_{i}(x)-\eta\right)+\frac{1}{m} \eta$ is assigned to agent $i \in V$ for processing without sharing the information about the function with any other agent.

The distributed algorithm will use the Bregman-distance functions $B_{x}$ and $B_{\eta}$, as introduced in Section 3.2. In addition, we introduce another collection of Bregmandistance functions, one for each of the agents in order to handle the Lagrange multipliers for its constraint set $\left\{x \mid f_{i}(x) \leq \eta\right\}$. For this, for each $i \in V$, we let $B_{\mu, i}(\cdot, \cdot)$ be a Bregman-distance function associated with a strongly convex function $\omega_{\mu, i}: \mathbb{R} \rightarrow \mathbb{R}$ with parameter $\sigma_{\mu, i}>0$.

The proposed primal-dual distributed algorithm for finding a saddle-point of the Lagrangian is as follows. At every iteration $k$, each agent $i$ has estimates $x_{k}^{i}, \eta_{k}^{i}$ and $\mu_{i}^{k}$, respectively, for an optimal solution of the min-max problem, the optimal value and the Lagrangian multiplier associated with the constraint $\left\{x \mid f_{i}(x) \leq \eta\right\}$ that agent $i$ is responsible for. Every agent firstly performs an intermittent adjustment of the variables $x_{k}^{i}$ and $\eta_{k}^{i}$ as in (10) to obtain the estimates $\tilde{x}_{k}^{i}$ and $\tilde{\eta}_{i}^{k}$. For convenience, we restate these updates:

$$
\left[\begin{array}{c}
\tilde{x}_{k}^{i} \\
\tilde{\eta}_{k}^{i}
\end{array}\right]=\sum_{j=1}^{m}\left[W_{k}\right]_{i j}\left[\begin{array}{c}
x_{k}^{j} \\
\eta_{k}^{j}
\end{array}\right] .
$$


This step ensures that agents locally align the variables that are coupling. We note that the agents do not have an intermittent adjustment for their multiplier estimates $\mu_{k}^{i}$, as these variables do not couple.

Then, every agent $i \in V$ generates new iterates by taking step toward minimizing its own Lagrangian function $L_{i}\left(x, \eta, \mu_{i}\right)=\mu_{i}\left(f_{i}(x)-\eta\right)+\frac{1}{m} \eta$ with respect to $(x, \eta)$ and maximizing it with respect to $\mu_{i}$, in the following manner:

$$
\begin{aligned}
x_{k+1}^{i} & =\operatorname{argmin}_{y \in X}\left[\alpha_{k} \mu_{k}^{i}\left\langle d_{k}^{i}+\varepsilon_{k}^{i}, y\right\rangle+B_{x}\left(y, \tilde{x}_{k}^{i}\right)\right], \\
\eta_{k+1}^{i} & =\operatorname{argmin}_{s \in D}\left[\alpha_{k}\left(1 / m-\mu_{k}^{i}\right) s+B_{\eta}\left(s, \tilde{\eta}_{k}^{i}\right)\right], \\
\mu_{k+1}^{i} & =\operatorname{argmin}_{\zeta \in I}\left[\alpha_{k}\left(\tilde{\eta}_{k}^{i}-f_{i}\left(\tilde{x}_{k}^{i}\right)\right) \zeta+B_{\mu, i}\left(\zeta, \mu_{k}^{i}\right)\right],
\end{aligned}
$$

where $d_{k}^{i}$ is a subgradient of $f_{i}(x)$ evaluated at $\tilde{x}_{k}^{i}$ and $\varepsilon_{k}$ is a random error in the subgradient evaluation. For each $i$, the initial values $x_{0}^{i} \in X, \eta_{0}^{i} \in D$, and $\mu_{0}^{i} \in I$ are random and independent from the stochastic errors $\varepsilon_{k}$.

\subsection{Algorithm Convergence}

The analysis is similar to that of algorithm (10)-(11) using exact-penalty approach. First, we state a lemma which relates the local iterates of the primal-dual algorithm to their respective average trajectory for the coupling variables $x_{k}^{i}$ and $\eta_{k}^{i}$.

Lemma 6. Let Assumptions 1 and 2 hold, and let the step sizes satisfy $\sum_{k=0}^{\infty} \alpha_{k}^{2}<\infty$. Then, for the instantaneous averages $\hat{x}_{k}$ and $\hat{\eta}_{k}$ of the iterates generated by algorithm (37)-(38), we have $\sum_{k=0}^{\infty} \alpha_{k}\left\|\hat{x}_{k}-x_{k}^{i}\right\|<\infty$ and $\sum_{k=0}^{\infty} \alpha_{k}\left|\hat{\eta}_{k}-\eta_{k}^{i}\right|<\infty$ for all $i \in V$, with probability 1 .

Proof. The proof is similar to that of Lemma 4 and uses relations $\mu_{k}^{i} \in I, i \in V$.

As a Lyapunov function, we choose the composite function

$$
\mathbf{B}\left(z, \mu, \mathbf{z}_{k}, \mu_{k}\right)=\sum_{i=1}^{m}\left[B_{x}\left(x, x_{k}^{i}\right)+B_{\eta}\left(\eta, \eta_{k}^{i}\right)+B_{\mu, i}\left(\mu_{i}, \mu_{k}^{i}\right)\right],
$$

where $\mathbf{z}_{k}=\left(x_{k}^{1}, \ldots, x_{k}^{m}, \eta_{k}^{1}, \ldots, \eta_{k}^{m}\right)$ and $\mu_{k}=\left(\mu_{k}^{1}, \ldots, \mu_{k}^{m}\right)$. We next establish a descent-type relation for the expected value of the Lyapunov function, which requires an appropriate sigma-field. We define the $\sigma$-field as follows:

$$
F_{k}=\left\{x_{t}^{i}, \eta_{t}^{i}, \mu_{t}^{i}, i \in V, t=0,1, \ldots, k\right\} \quad \text { for } k \geq 0 .
$$

From now on, we abbreviate the conditional expectation notation by $\mathbb{E}_{k}[\cdot]=\mathbb{E}\left[\cdot \mid F_{k}\right]$. We have the following result that will play the key role in the convergence analysis.

Lemma 7. Let $D \subset \mathbb{R}$ be a convex compact set such that $\eta^{*} \in D$. Let Assumptions 1 , 2 and 3 hold. Then, for algorithm (37)-(38) the following relation holds with probability 1 , for all $k \geq 0$, all $z^{*}=\left(x^{*}, \eta^{*}\right)$ with $x^{*} \in X^{*}$, and all optimal multipliers $\mu^{*}$, 


$$
\begin{aligned}
& \mathbb{E}_{k}\left[\boldsymbol{B}\left(z^{*}, \mu^{*}, z_{k+1}, \mu_{k+1}\right)\right] \leq \boldsymbol{B}\left(z^{*}, \mu^{*}, z_{k}, \mu_{k}\right)+2 \alpha_{k} \sum_{j=1}^{m}\left(C\left\|x_{k}^{j}-\hat{x}_{k}\right\|+\left|\eta_{k}^{j}-\hat{\eta}_{k}\right|\right) \\
& -\alpha_{k}\left(\mathscr{L}\left(\hat{x}_{k}, \hat{\eta}_{k}, \mu^{*}\right)-\mathscr{L}\left(x^{*}, \eta^{*}, \mu_{k}\right)\right) \\
& +\alpha_{k}^{2}\left(m \frac{(C+v)^{2}}{2 \sigma_{x}}+\sum_{i=1}^{m} \frac{\left(1 / m-\mu_{k}^{i}\right)^{2}}{2 \sigma_{\eta}}+\sum_{i=1}^{m} \frac{\left(f_{i}\left(\tilde{x}_{k}^{i}\right)-\tilde{\eta}_{k}^{i}\right)^{2}}{2 \sigma_{\mu, i}}\right) .
\end{aligned}
$$

Proof. Let $z=\left(x^{*}, \eta^{*}\right) \in X^{*} \times D$ and $\mu^{*} \in I^{m}$ be arbitrary optimal primal-dual pairs, where $I^{m}$ is the product of $m$ copies of the interval $I=[0,1]$. Using the optimality conditions for (38) and proceeding as in the proof of Lemma 5, we obtain with probability 1 ,

$$
\mathbb{E}_{k}\left[B_{x}\left(x^{*}, x_{k+1}^{i}\right)\right] \leq B_{x}\left(x^{*}, \tilde{x}_{k}^{i}\right)-\alpha_{k} \mu_{k}^{i}\left\langle\mathbb{E}_{k}\left[d_{k}^{i}+\varepsilon_{k}^{i}\right], \tilde{x}_{k}^{i}-x^{*}\right\rangle+\alpha_{k}^{2} \frac{(C+v)^{2}}{2 \sigma_{x}} .
$$

Now, since $\mathbb{E}_{k}\left[\varepsilon_{k}^{i}\right]=0$, and $d_{k}^{i}$ is a subgradient of $f_{i}(x)$ at $\tilde{x}_{k}^{i}$ it follows that

$$
\mathbb{E}_{k}\left[B_{x}\left(x^{*}, x_{k+1}^{i}\right)\right] \leq B_{x}\left(x^{*}, \tilde{x}_{k}^{i}\right)-\alpha_{k} \mu_{k}^{i}\left(f_{i}\left(\tilde{x}_{k}^{i}\right)-f_{i}\left(x^{*}\right)\right)+\alpha_{k}^{2} \frac{(C+v)^{2}}{2 \sigma_{x}} .
$$

A similar analysis gives the following inequality for the iterates $\eta_{k}^{i}$,

$$
B_{\eta}\left(\eta^{*}, \eta_{k+1}^{i}\right) \leq B_{\eta}\left(\eta^{*}, \tilde{\eta}_{k}^{i}\right)-\alpha_{k}\left(1 / m-\mu_{k}^{i}\right)\left(\tilde{\eta}_{k}^{i}-\eta^{*}\right)+\alpha_{k}^{2} \frac{\left(1 / m-\mu_{k}^{i}\right)^{2}}{2 \sigma_{\eta}},
$$

and the inequality for the multiplier iterates $\mu_{k}^{i}$,

$$
B_{\mu, i}\left(\mu_{i}^{*}, \mu_{k+1}^{i}\right) \leq B_{\mu, i}\left(\mu_{i}^{*}, \mu_{k}^{i}\right)+\alpha_{k}\left(f_{i}\left(\tilde{x}_{k}^{i}\right)-\tilde{\eta}_{k}^{i}\right)\left(\mu_{k}^{i}-\mu_{i}^{*}\right)+\alpha_{k}^{2} \frac{\left(f_{i}\left(\tilde{x}_{k}^{i}\right)-\tilde{\eta}_{k}^{i}\right)^{2}}{2 \sigma_{\mu, i}} .
$$

Summing equations (40)-(42), and then summing the resulting relation over all $i \in V$, we have with probability 1 ,

$$
\begin{aligned}
& \mathbb{E}_{k}\left[\mathbf{B}\left(z^{*}, \mu^{*}, \mathbf{z}_{k+1}, \mu_{k+1}\right)\right] \leq \mathbf{B}\left(z^{*}, \mu^{*}, \tilde{\mathbf{z}}_{k}, \mu_{k}\right)+\alpha_{k}^{2} K \\
& -\alpha_{k} \sum_{i=1}^{m}(\underbrace{\mu_{k}^{i}\left(f_{i}\left(\tilde{x}_{k}^{i}\right)-f_{i}\left(x^{*}\right)\right)}_{\text {Term } 1}+\underbrace{\left(1 / m-\mu_{k}^{i}\right)\left(\tilde{\eta}_{k}^{i}-\eta^{*}\right)}_{\text {Term } 2}-\underbrace{\left(f_{i}\left(\tilde{x}_{k}^{i}\right)-\tilde{\eta}_{k}^{i}\right)\left(\mu_{k}^{i}-\mu_{i}^{*}\right)}_{\text {Term } 3}),
\end{aligned}
$$

where we use notation (39) for the Lyapunov function, $\tilde{\mathbf{z}}_{k}=\left(\tilde{x}_{k}^{1}, \ldots, \tilde{x}_{k}^{m}, \tilde{\eta}_{k}^{1}, \ldots, \tilde{\eta}_{k}^{m}\right)$, and $K=m \frac{(C+v)^{2}}{2 \sigma_{x}}+\sum_{i=1}^{m} \frac{\left(1 / m-\mu_{k}^{i}\right)^{2}}{2 \sigma_{\eta}}+\sum_{i=1}^{m} \frac{\left(f_{i}\left(\tilde{x}_{k}^{i}\right)-\tilde{\eta}_{k}^{i}\right)^{2}}{2 \sigma_{\mu, i}}$. We now estimate the identified terms in the preceding relation by adding and subtracting $f\left(\hat{x}_{k}\right)$ or $\hat{\eta}_{k}$. We have $f_{i}\left(x_{k}^{i}\right)-f_{i}\left(x^{*}\right)=\left(f_{i}\left(\hat{x}_{k}\right)-f_{i}\left(x^{*}\right)\right)+\left(f_{i}\left(\tilde{x}_{k}^{i}\right)-f_{i}\left(\hat{x}_{k}\right)\right) \geq f_{i}\left(\hat{x}_{k}\right)-f_{i}\left(x^{*}\right)-C\left\|\tilde{x}_{k}^{i}-\hat{x}_{k}\right\|$, 
where the inequality follows from the convexity of $f_{i}$ and subgradient boundedness. Since $\mu_{k}^{i} \in I=[0,1]$ for all $i \in V$, it follows

$$
\operatorname{Term} 1 \geq \mu_{k}^{i}\left(f_{i}\left(\hat{x}_{k}\right)-f_{i}\left(x^{*}\right)\right)-C\left\|\tilde{x}_{k}^{i}-\hat{x}_{k}\right\| .
$$

For the second term, we have

$$
\begin{aligned}
\left(1 / m-\mu_{k}^{i}\right)\left(\tilde{\eta}_{k}^{i}-\eta^{*}\right) & =\left(1 / m-\mu_{k}^{i}\right)\left(\hat{\eta}_{k}-\eta^{*}\right)+\left(1 / m-\mu_{k}^{i}\right)\left(\tilde{\eta}_{k}^{i}-\hat{\eta}_{k}\right) \\
& \geq\left(1 / m-\mu_{k}^{i}\right)\left(\hat{\eta}_{k}-\eta^{*}\right)-\left|1 / m-\mu_{k}^{i}\right|\left|\tilde{\eta}_{k}^{i}-\hat{\eta}_{k}\right| .
\end{aligned}
$$

Using $\mu_{k}^{i} \in I=[0,1]$ for all $i \in V$, we obtain

$$
\operatorname{Term} 2 \geq\left(1 / m-\mu_{k}^{i}\right)\left(\hat{\eta}_{k}-\eta^{*}\right)-\left|\tilde{\eta}_{k}^{i}-\hat{\eta}_{k}\right| .
$$

For the third term, we write

$$
\begin{aligned}
\left(f_{i}\left(\tilde{x}_{k}^{i}\right)-\tilde{\eta}_{k}^{i}\right)\left(\mu_{k}^{i}-\mu_{i}^{*}\right)= & \left(f_{i}\left(\hat{x}_{k}\right)-\hat{\eta}_{k}\right)\left(\mu_{k}^{i}-\mu_{i}^{*}\right) \\
& +\left(\left(f_{i}\left(\tilde{x}_{k}^{i}\right)-f_{i}\left(\hat{x}_{k}\right)\right)-\left(\tilde{\eta}_{k}^{i}-\hat{\eta}_{k}\right)\right)\left(\mu_{k}^{i}-\mu_{i}^{*}\right) \\
\geq & \left(f_{i}\left(\hat{x}_{k}\right)-\hat{\eta}_{k}\right)\left(\mu_{k}^{i}-\mu_{i}^{*}\right)-\left(C\left\|\tilde{x}_{k}^{i}-\hat{x}_{k}\right\|+\left|\tilde{\eta}_{k}-\hat{\eta}_{k}\right|\right)\left|\mu_{k}^{i}-\mu_{i}^{*}\right|,
\end{aligned}
$$

where the inequality follows by the convexity of $f_{i}$ and the subgradient boundedness. Again, since $\mu_{k}^{i}, \mu \in I$, we see that $\left|\mu_{k}^{i}-\mu_{i}\right| \leq 1$, implying

$$
\text { Term } 3 \geq\left(f_{i}\left(\hat{x}_{k}\right)-\hat{\eta}_{k}\right)\left(\mu_{k}^{i}-\mu_{i}^{*}\right)-\left(C\left\|\tilde{x}_{k}^{i}-\hat{x}_{k}\right\|+\left|\tilde{\eta}_{k}-\hat{\eta}_{k}\right|\right) .
$$

Substituting estimates (44)-(46) back in relation (43), we obtain

$$
\begin{aligned}
& \mathbb{E}_{k}\left[\mathbf{B}\left(z^{*}, \mu^{*}, \mathbf{z}_{k+1}, \mu_{k+1}\right)\right] \leq \mathbf{B}\left(z^{*}, \mu^{*}, \tilde{\mathbf{z}}_{k}, \mu_{k}\right)+\alpha_{k}^{2} K+2 \alpha_{k} \sum_{i=1}^{m}\left(C\left\|\tilde{x}_{k}^{i}-\hat{x}_{k}\right\|+\left|\tilde{\eta}_{k}^{i}-\hat{\eta}_{k}\right|\right) \\
& -\alpha_{k} \sum_{i=1}^{m}\left(\mu_{k}^{i}\left(f_{i}\left(\hat{x}_{k}\right)-f_{i}\left(x^{*}\right)\right)+\left(1 / m-\mu_{k}^{i}\right)\left(\hat{\eta}_{k}-\eta^{*}\right)-\left(f_{i}\left(\hat{x}_{k}\right)-\hat{\eta}_{k}\right)\left(\mu_{k}^{i}-\mu_{i}^{*}\right)\right) .
\end{aligned}
$$

By definition in (10), the estimates $\tilde{x}_{k}^{i}$ and $\tilde{\eta}_{k}^{i}$ are convex combinations of $x_{k}^{j}$ and $\eta_{k}^{j}$, respectively. Since under our assumption the Bregman-distance functions $B_{x}$ and $B_{\eta}$ are convex in the second argument, it follows

$$
B_{x}\left(x^{*}, \tilde{x}_{k}^{i}\right) \leq \sum_{j=1}^{m}\left[W_{k}\right]_{i j} B_{x}\left(x^{*}, x_{k}^{j}\right), \quad B_{\eta}\left(\eta^{*}, \tilde{\eta}_{k}^{i}\right) \leq \sum_{j=1}^{m}\left[W_{k}\right]_{i j} B_{\eta}\left(\eta^{*}, \eta_{k}^{j}\right) .
$$

By summing these relations over $i \in V$ and using the doubly stochasticity of the weight matrix $W_{k}$, we obtain

$$
\sum_{i=1}^{m} B_{x}\left(x^{*}, \tilde{x}_{k}^{i}\right) \leq \sum_{j=1}^{m} B_{x}\left(x^{*}, x_{k}^{j}\right), \quad \sum_{i=1}^{m} B_{\eta}\left(\eta^{*}, \tilde{\eta}_{k}^{i}\right) \leq \sum_{j=1}^{m} B_{\eta}\left(\eta^{*}, \eta_{k}^{j}\right) .
$$


Using the definition of $\mathbf{B}$ (see (39)) and these relations, from (47) we have

$$
\begin{gathered}
\mathbb{E}_{k}\left[\mathbf{B}\left(z^{*}, \mu^{*}, \mathbf{z}_{k+1}, \mu_{k+1}\right)\right] \leq \mathbf{B}\left(z^{*}, \mu^{*}, \mathbf{z}_{k}, \mu_{k}\right)+\alpha_{k}^{2} K+2 \alpha_{k} \sum_{i=1}^{m}\left(C\left\|\tilde{x}_{k}^{i}-\hat{x}_{k}\right\|+\left|\tilde{\eta}_{k}^{i}-\hat{\eta}_{k}\right|\right) \\
-\alpha_{k} \underbrace{\sum_{i=1}^{m}\left(\mu_{k}^{i}\left(f_{i}\left(\hat{x}_{k}\right)-f_{i}\left(x^{*}\right)\right)+\left(1 / m-\mu_{k}^{i}\right)\left(\hat{\eta}_{k}-\eta^{*}\right)-\left(f_{i}\left(\hat{x}_{k}\right)-\hat{\eta}_{k}\right)\left(\mu_{k}^{i}-\mu_{i}^{*}\right)\right)}_{\text {Term }} .
\end{gathered}
$$

Now, we consider the identified term in (48). We note that

$$
\begin{gathered}
\sum_{i=1}^{m} \mu_{k}^{i}\left(f_{i}\left(\hat{x}_{k}\right)-f_{i}\left(x^{*}\right)\right)+\left(1 / m-\mu_{k}^{i}\right)\left(\hat{\eta}_{k}-\eta^{*}\right)=\mathscr{L}\left(\hat{x}_{k}, \hat{\eta}_{k}, \mu_{k}\right)-\mathscr{L}\left(x^{*}, \eta^{*}, \mu_{k}\right), \\
\sum_{i=1}^{m}\left(f_{i}\left(\hat{x}_{k}\right)-\hat{\eta}_{k}\right)\left(\mu_{k}^{i}-\mu_{i}^{*}\right)=\mathscr{L}\left(\hat{x}_{k}, \hat{\eta}_{k}, \mu_{k}\right)-\mathscr{L}\left(\hat{x}_{k}, \hat{\eta}_{k}, \mu^{*}\right),
\end{gathered}
$$

which imply

$$
\text { Term }=\mathscr{L}\left(\hat{x}_{k}, \hat{\eta}_{k}, \mu^{*}\right)-\mathscr{L}\left(\hat{x}^{*}, \eta^{*}, \mu_{k}\right) .
$$

Furthermore, from convexity of the norm and the absolute value functions, since $W_{k}$ is doubly stochastic, and $\tilde{x}_{k}^{i}=\sum_{j=1}^{m} x_{k}^{j}$ and $\tilde{\eta}_{k}^{i}=\sum_{j=1}^{m} x_{k}^{j}$ it follows that

$$
\sum_{i=1}^{m}\left(C\left\|\tilde{x}_{k}^{i}-\hat{x}_{k}\right\|+\left|\tilde{\eta}_{k}^{i}-\hat{\eta}_{k}\right|\right) \leq \sum_{j=1}^{m}\left(C\left\|x_{k}^{j}-\hat{x}_{k}\right\|+\left|\eta_{k}^{j}-\hat{\eta}_{k}\right|\right) \text {. }
$$

Using (49)-(50) and $K=m \frac{(C+v)^{2}}{2 \sigma_{x}}+\sum_{i=1}^{m} \frac{\left(1 / m-\mu_{k}^{i}\right)^{2}}{2 \sigma_{\eta}}+\sum_{i=1}^{m} \frac{\left(f_{i}\left(\tilde{x}_{k}^{i}\right)-\tilde{\eta}_{k}^{i}\right)^{2}}{2 \sigma_{\mu, i}}$, from (48) we obtain the desired relation.

In order to connect the limiting vector $(\bar{x}, \bar{\eta}, \bar{\mu})$ of the iterates generated by the primal-dual algorithm to the solutions of problem (34), we will invoke the necessary and sufficient Karush-Khun-Tucker (KKT) optimality conditions. These conditions are stated below for convenience, as adjusted to our problem.

Theorem 4. The vector $(\bar{x}, \bar{\eta}, \bar{\mu})$ is a primal-dual optimal vector if and only if $f_{i}(\bar{x}) \leq \bar{\eta}$ and $\bar{\mu} \in I^{m}$ and the following two conditions are satisfied

$$
(\bar{x}, \bar{\eta}, \bar{\mu}) \in \operatorname{argmin}_{(x, \eta) \in X \times D} \mathscr{L}(x, \eta, \bar{\mu}) \quad \bar{\mu} \in \operatorname{argmax}_{\mu \in I^{m}} \mathscr{L}(\bar{x}, \bar{\eta}, \mu) .
$$

We are now in position to assert the convergence property of the algorithm for a diminishing step size.

Theorem 5. Let Assumptions 1, 2, and 3 hold, except for subgradient norm boundedness of Assumption 2-b. Assume that $X$ and $D$ are compact convex sets, and that min-max problem (3) has a unique optimal solution $x^{*}$. Let the step sizes satisfy $\sum_{k=0}^{\infty} \alpha_{k}=\infty$ and $\sum_{k=0}^{\infty} \alpha_{k}^{2}<\infty$. Then, the agents' iterates $x_{k}^{i}, \eta_{k}^{i}$, $\mu_{k}^{i}$, generated by algorithm (37)-(38) are such that with probability 1 for all $i \in V$ : 
(a) The estimates $x_{k}^{i}$ converge to the optimal point $x^{*}$.

(b) The estimates $\eta_{k}^{i}$ converge to the optimal value $\eta^{*}$ of the min-max problem.

(c) The dual iterates $\mu_{k}^{i}$ converge to a (random) optimal dual variable $\mu_{i}^{*}$.

Proof. The proof proceeds by applying the Robbins-Siegmund result (Theorem 2) to the relation of Lemma 7. By our assumption on the step sizes we have $\sum_{k=0}^{\infty} \alpha_{k}^{2}<$ $\infty$, which immediately yields $\sum_{k=0}^{\infty} \alpha_{k}^{2} m \frac{(C+v)^{2}}{2 \sigma_{x}}<\infty$. Since, the projection step in algorithm (38) constrains the dual variables $\mu_{k}^{i}$ to lie in the interval $[0,1]$, it follows that $\sum_{k=0}^{\infty} \alpha_{k}^{2} \sum_{i=1}^{m} \frac{\left(1 / m-\mu_{k}^{i}\right)^{2}}{2 \sigma_{\eta}}<\infty$ with probability 1 . Moreover, as the constraint set $X$ and the set $D$ are compact, and $f_{i}$ are continuous, the term $\left(f_{i}\left(\tilde{x}_{k}^{i}\right)-\tilde{\eta}_{k}^{i}\right)^{2}$ is uniformly bounded for all $i$ and $k$, implying that $\sum_{k=0}^{\infty} \alpha_{k}^{2} \sum_{i=1}^{m} \frac{\left(f_{i}\left(x_{k}^{i}\right)-\tilde{\eta}_{k}^{i}\right)^{2}}{2 \sigma_{\mu, i}}<\infty$ with probability 1. From Lemma 6 we have $\sum_{k=0}^{\infty} \alpha_{k}\left(C \sum_{j=1}^{m}\left\|\hat{x}_{k}-x_{k}^{j}\right\|+\left|\hat{\eta}_{k}-\eta_{k}^{j}\right|\right)<\infty$ with probability 1 . As $\sum_{k=0}^{\infty} \alpha_{k}=\infty$, it follows that with probability 1 ,

$$
\lim _{k \rightarrow \infty} \sum_{j=1}^{m}\left(\left\|\hat{x}_{k}-x_{k}^{j}\right\|+\left|\hat{\eta}_{k}-\eta_{k}^{j}\right|\right)=0 .
$$

By the saddle-point Theorem 1 we have $\mathscr{L}\left(\hat{x}_{k}, \hat{\eta}_{k}, \mu^{*}\right)-\mathscr{L}\left(x^{*}, \eta^{*}, \mu_{k}\right) \geq 0$. Thus, all the conditions of Theorem 2 are satisfied.

By applying Theorem 2 we infer that $\mathbf{B}\left(z^{*}, \mu^{*}, \mathbf{z}_{k}, \mu_{k}\right)$ converges for every dualoptimal $\mu^{*}$ with probability 1 , and that $\sum_{k=0}^{\infty} \alpha_{k}\left(\mathscr{L}\left(\hat{x}_{k}, \hat{\eta}_{k}, \mu^{*}\right)-L\left(x^{*}, \eta^{*}, \mu_{k}\right)\right)<\infty$ also holds with probability 1 . Since $\sum_{k=0}^{\infty} \alpha_{k}=\infty$, it follows that with probability 1 ,

$$
\lim _{k \rightarrow \infty}\left(\mathscr{L}\left(\hat{x}_{k}, \hat{\eta}_{k}, \mu^{*}\right)-L\left(x^{*}, \eta^{*}, \mu_{k}\right)\right)=0 .
$$

As the sequences $\left.\left\{x_{k}^{i}\right\}, \eta_{k}^{i}\right\},\left\{\mu_{k}^{i}\right\}$ are bounded, they have accumulation points in the sets $X, D$ and $I=[0,1]$, respectively. We can use Cantor diagonalization-type argument to select a subsequence $\left\{k_{\ell}\right\}$ along which the following relations hold with probability 1:

$$
\begin{gathered}
\lim _{\ell \rightarrow \infty}\left(\hat{x}_{k_{\ell}}, \hat{\eta}_{k_{\ell}}, \mu_{k_{\ell}}\right)=(\bar{x}, \bar{\eta}, \bar{\mu}) \quad \text { with } \quad(\bar{x}, \bar{\eta}, \bar{\mu}) \in X \times D \times I^{m}, \\
\lim _{\ell \rightarrow \infty}\left(\mathscr{L}\left(\hat{x}_{k_{\ell}}, \hat{\eta}_{k_{\ell}}, \mu^{*}\right)-\mathscr{L}\left(x^{*}, \eta^{*}, \mu_{k_{\ell}}\right)\right)=0, \\
\lim _{\ell \rightarrow \infty}\left\|\hat{x}_{k_{\ell}}-x_{k_{\ell}}^{j}\right\|=0, \quad \lim _{\ell \rightarrow \infty}\left|\hat{\eta}_{k_{\ell}}-\eta_{k_{\ell}}^{j}\right|=0 \quad \text { for all } j \in V .
\end{gathered}
$$

We now examine the consequences of these relations. Since $\left(x^{*}, \eta^{*}, \mu^{*}\right)$ is a saddlepoint of the Lagrangian, it follows that $\mathscr{L}\left(\bar{x}, \bar{\eta}, \mu^{*}\right) \geq \mathscr{L}\left(x^{*}, \eta^{*}, \mu^{*}\right) \geq \mathscr{L}\left(x^{*}, \eta^{*}, \bar{\mu}\right)$, implying that both inequalities hold as equalities. Therefore,

$$
(\bar{x}, \bar{\eta}) \in \operatorname{argmin}_{(x, \eta) \in X \times D} \mathscr{L}\left(x, \eta, \mu^{*}\right), \quad \bar{\mu} \in \operatorname{argmax}_{\mu \in I^{m}} \mathscr{L}\left(x^{*}, \eta^{*}, \mu\right) .
$$


Since $\left(x^{*}, \eta^{*}\right)$ is the unique solution of problem (34) (by our assumption), the preceding relations together with the KKT conditions (Theorem 4) imply that $(\bar{x}, \bar{\eta})=\left(x^{*}, \eta^{*}\right)$ and that $\bar{\mu}$ is an optimal dual multiplier.

It remains to show that the whole sequences converge to the desired optimal points. From (53) it follows that $\lim _{\ell \rightarrow \infty}\left\|x_{k_{\ell}}^{j}-x^{*}\right\|=0$ and $\lim _{\ell \rightarrow \infty}\left|\eta_{k_{\ell}}^{j}-\eta^{*}\right|=0$ with probability 1 for all $j$, implying $\lim _{\ell \rightarrow \infty} \mathbf{B}\left(z^{*}, \bar{\mu}, \mathbf{z}_{k_{\ell}}, \mu_{k_{\ell}}\right)=0$. However, we have established that the sequence $\mathbf{B}\left(z^{*}, \mu^{*}, \mathbf{z}_{k}, \mu_{k}\right)$ converges with probability 1 for any optimal dual vector $\mu^{*}$, so it converges with $\mu^{*}=\bar{\mu}$. Thus, it follows $\lim _{k \rightarrow \infty} \mathbf{B}\left(z^{*}, \bar{\mu}, \mathbf{z}_{k}, \mu_{k}\right)=0$ with probability 1 . Owing to the strong convexity of the Bregman functions we have

$$
\mathbf{B}\left(z^{*}, \bar{\mu}, \mathbf{z}_{k}, \mu_{k}\right) \geq \sum_{i=1}^{m}\left(\frac{\sigma_{x}}{2}\left\|x^{*}-x_{k}^{i}\right\|^{2}+\frac{\sigma_{\eta}}{2}\left|\eta^{*}-\eta_{k}^{i}\right|^{2}+\frac{\sigma_{\mu, i}}{2}\left|\bar{\mu}_{i}-\mu_{k}^{i}\right|^{2}\right),
$$

which yields $x_{k}^{i} \rightarrow x^{*}, \eta_{k}^{i} \rightarrow \eta^{*}$ and $\mu_{k}^{i} \rightarrow \bar{\mu}_{i}$ with probability 1 for all $i \in V$.

\section{Min-Max Game Against Exogenous Player}

In this section we consider a different formulation than the one discussed so far. We consider the case when the network of cooperative agents need to solve a min-max game against an exogenous player. The exogenous player is a malicious agent/nature which adversely affects the cost of each agent. Let us denote the action of the adversarial agent by $\xi$. We also require that the feasible set of allowable actions $\xi$ is a compact set denoted by $\Theta$. The objective is to solve:

$$
\min _{x \in X} \max _{\xi \in \Theta} \sum_{i=1}^{m} f_{i}(x, \xi)
$$

This can be thought of as the robust version of the problem considered in [35, 29], where the optimization problem of the form $\min _{x \in X} \sum_{i=1}^{m} \mathbb{E}_{\xi}\left[f_{i}(x, \xi)\right]$ was considered. In certain cases when it is desired to model the unknown signal $\xi$ as lying in an uncertainty set $\Theta$ the robust version of problem (54) is more suitable. The problem (54) could alternatively be thought of as a zero sum game between the exogenous player and the network. To guarantee the existence of a min-max optimal solution to (54) we impose the following assumption.

\section{Assumption 4 Let the following hold:}

(a) The functions $f_{i}$ are continuous over some open set containing $X \times \Theta$.

(b) The cost functions $f_{i}(x, \xi)$ are convex in $x$ for every fixed value of $\xi \in \Theta$, and concave in $\xi$ for every fixed $x \in X$.

(c) The constraint sets $X$ and $\Theta$ are convex and compact.

Under Assumption 4 the min-max problem (54) admits a solution set $X^{*} \times \Theta^{*}$ such that for any $x^{*} \in X^{*}$ and $\xi^{*} \in \Theta^{*}$, we have the saddle-point property [4]: 


$$
\sum_{i=1}^{m} f_{i}\left(x^{*}, \xi\right) \leq \sum_{i=1}^{m} f_{i}\left(x^{*}, \xi^{*}\right) \leq \sum_{i=1}^{m} f_{i}\left(x, \xi^{*}\right) \quad \text { for all } x \in X \text { and } \xi \in \Theta .
$$

Let $B_{x}(\cdot, \cdot)$ and $B_{\xi}(\cdot, \cdot)$ be Bregman-distance functions for the sets $X$ and $\Theta$, respectively. We propose the following algorithm for min-max problem (54): at each iteration, at first, the agents update using the estimates $x_{k}^{j}$ and $\xi_{k}^{i}$ and obtain intermittent estimates

$$
\left[\begin{array}{c}
\tilde{x}_{k}^{i} \\
\tilde{\xi}_{k}^{i}
\end{array}\right]=\sum_{j=1}^{m}\left[W_{k}\right]_{i j}\left[\begin{array}{c}
x_{k}^{j} \\
\xi_{k}^{j}
\end{array}\right],
$$

where $W_{k}$ is a weight matrix as in (10). Using these intermittent adjustment, every agent updates according to the following rules:

$$
\begin{aligned}
& x_{k+1}^{i}=\operatorname{argmin}_{y \in X}\left[\alpha_{k}\left\langle\nabla_{x} f_{i}\left(\tilde{x}_{k}^{i}, \tilde{\xi}_{k}^{i}\right), y\right\rangle+B_{x}\left(y, \tilde{x}_{k}^{i}\right)\right], \\
& \xi_{k+1}^{i}=\operatorname{argmin}_{\zeta \in \Theta}\left[-\alpha_{k}\left\langle\nabla_{\xi} f_{i}\left(\tilde{x}_{k}^{i}, \tilde{\xi}_{k}^{i}\right), \zeta\right\rangle+B_{\xi}\left(\zeta, \tilde{\xi}_{k}^{i}\right)\right],
\end{aligned}
$$

where $\nabla_{x}$ and $\nabla_{\xi}$ denote the partial derivative operators with respect to the variables $x$ and $\xi$, respectively. The initial points $x_{0}^{i}$ and $\xi_{0}^{i}$ satisfy $x_{0}^{i} \in X$ and $\xi_{0}^{i} \in \Theta$ for all $i$. It is also assumed that the constraint sets $X$ and $\Theta$ are common knowledge for all agents $i \in V$.

The analysis of the algorithm follows along lines similar to that of the primaldual algorithm (37)-(38). This can be seen in light of the fact that the primal-dual algorithm computes a saddle-point of the Lagrangian function in (5), whereas algorithm (56)-(57) computes a saddle-point of problem (54). A major difference between the algorithms is the fact that in (38) the agents update their own local dual variables $\mu_{k}^{i}$, whereas in the algorithm (57) the agents update the whole vector $\xi$ which is coupling the agents. Note that there is no stochasticity in the current formulation unless we consider a stochastic model of the network. The final result is that asymptotically the agents estimates $x_{k}^{i}$ and $\xi_{k}^{i}$ converge to a common min-max optimal pair $\left(x^{*}, \xi^{*}\right)$. We formalize the statement in the following theorem.

Theorem 6. Let Assumption 1 and 4 hold. Assume that problem (54) has an optimal set of the form $\left\{x^{*}\right\} \times \Theta^{*}$. Moreover, assume that the Bregman-distance functions $B_{x}(y, v)$ and $B_{\xi}(\zeta, \phi)$ are convex in their second arguments $v$ and $\phi$, respectively for every fixed $y$ and $\zeta$. If the step sizes $\alpha_{k}$ in algorithm (56)-(57) are chosen to satisfy $\sum_{k=0}^{\infty} \alpha_{k}=\infty$ and $\sum_{k=0}^{\infty} \alpha_{k}^{2}<\infty$, then the local variables $\left(x_{k}^{i}, \xi_{k}^{i}\right)$ converge to a common saddle-point solution $\left(x^{*}, \xi^{*}\right)$ of the min-max problem (54), for all $i \in V$.

Proof. The proof is similar to the proof of Theorem 5, with the Lyapunov function $\sum_{i=1}^{m}\left(B_{x}\left(x^{*}, x_{k}^{i}\right)+B_{\xi}\left(\xi^{*}, \xi_{k}^{i}\right)\right)$ for an arbitrary saddle-point solution $\left(x^{*}, \xi^{*}\right)$. 


\section{Uplink Power Control}

In this section we show the suitability of our algorithms in (10)-(11) and (37)-(38) to achieve a min-max fair allocation of utility in a cellular network. We will keep our discussion brief and refer the readers to [12] for a general discussion on the power allocation problem. We will be using the formulation discussed in [30].

There are $m$ mobile users (MU) in neighboring cells communicating with their respective base stations (BS) using a common wireless channel. Let $p_{i}$ denote the power used by MU $i$ to communicate with its base station. Due to the shared nature of the wireless medium the total received SINR at BS $i$ is given by

$$
\gamma_{i}\left(\bar{p}, \bar{h}_{i}\right)=\frac{p_{i} h_{i, i}^{2}}{\sigma_{i}^{2}+\sum_{j \neq i} p_{j} h_{i, j}^{2}},
$$

where $h_{i, j}$ is the channel coefficient between MU $j$ and BS $i$, and $\sigma_{i}^{2}$ is the receiver noise variance. The vector containing power variables $p_{i}$ is denoted $\bar{p}$ and the vector of channel coefficients at BS $i$ is denoted $\bar{h}_{i}$. The power variables are non-negative and constrained to a maximum value of $p_{t}$, i.e., $0 \leq p_{i} \leq p_{t}$ for all $i$.

Let $U_{i}\left(\gamma_{i}\left(\bar{p}, \bar{h}_{i}\right)\right)$ be the utility derived by BS $i$ and $V\left(p_{i}\right)$ be a cost function penalizing excessive power. We are interested in finding an allocation that minimizes the worst case loss to any agent $i$, which amounts to solving the following problem:

$$
\min _{\bar{p} \in \Pi} \max _{i \in V}\left[V\left(p_{i}\right)-U_{i}\left(\gamma_{i}\left(\bar{p}, \bar{h}_{i}\right)\right)\right]
$$

where $\Pi=\left\{\bar{p} \in \mathbb{R}^{m} \mid 0 \leq p_{i} \leq p_{t}\right.$ for all $\left.i\right\}$ and $p_{t}$ is the maximum power. We consider the $\log$ arithmic utility function $U_{i}(u)=\log (u)$ for $u>0$. Using the transformation $p_{i}=e^{x_{i}}$, it can be shown that the preceding problem can be cast in the form of (1), with a cost function for each base station $i$ given by:

$$
f_{i}(x)=\log \left(\sigma_{i}^{2} h_{i, i}^{-2} e^{-x_{i}}+\sum_{j \neq i} h_{i, i}^{-2} h_{j, i}^{2} e^{x_{j}-x_{i}}\right)+V\left(e^{x_{i}}\right),
$$

and $X=\left\{x \in \mathbb{R}^{m} \mid x_{i} \leq \log \left(p_{t}\right)\right.$ for all $\left.i\right\}$.

We have considered a cellular network of 16 square cells of the same size $(m=$ 16). The connectivity network for the BSs is shown in Figure 1. Within each cell, the MU is randomly located (with a uniform distribution over the cell) and the base station is located at the center of the cell. The channel coefficient $h_{i, j}$ is assumed to decay as the fourth power of the distance between the MU $j$ and the BS $i$. The shadow fading is assumed to be log-normal with variance 0.1 . The receiver noise variance $\sigma_{i}^{2}$ is taken to be 0.01 . The cost of the power is modeled as $V\left(p_{i}\right)=10^{-3} p_{i}$.

In the simulations, there are no stochastic errors, i.e., all gradients and subgradients are evaluated without stochastic errors. Four algorithms are used, namely, the standard (centralized) gradient descent algorithm (applied to the penalized problem), a centralized primal-dual algorithm (that computes a saddle point of the min- 


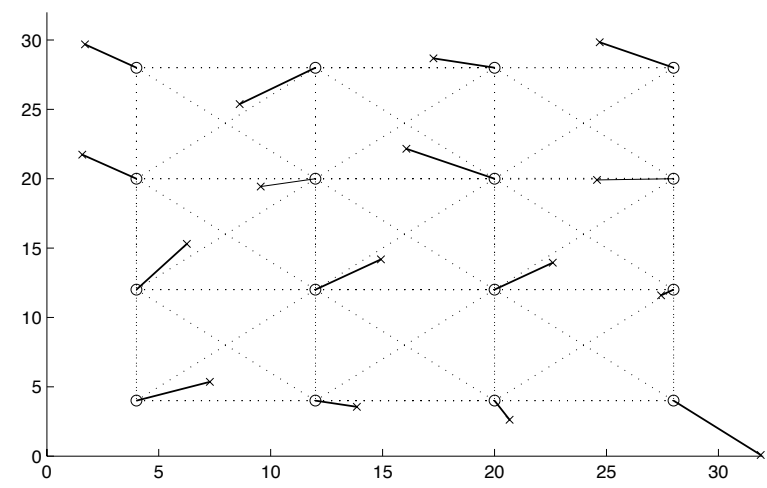

Fig. 1 The circles denote the BSs. The dotted lines denote the communication links between adjacent BSs. The crosses denote the MUs. The bold lines connect each MU to its respective BS.

max problem), the distributed exact penalty (10)-(11), and the distributed primaldual (37)-(38). The standard (centralized) gradient descent algorithm is used to determine the optimal min-max allocation, while the centralized primal-dual is used to get a sense of dual optimal variables. In the distributed algorithms, the weight matrices $W_{k}$ are all equal since the connectivity graph is static, i.e., $W_{k}=W$ for all $k$. The weights $W_{i j}$ are given by:

$$
W_{i j}=\frac{1}{\max \left\{\left|N_{i}\right|,\left|N_{j}\right|\right\}} \quad \text { if } i \text { and } j \text { are neighbors, }
$$

and otherwise $W_{i j}=0$, where $\left|N_{i}\right|$ denotes the cardinality of the neighbor set $N_{i}$ (which includes agent $i$ itself). The stepsize is $\alpha_{k}=\frac{10}{k}$ in the centralized methods, $\alpha_{k}=\frac{50}{k^{0.65}}$ in the distributed exact penalty method, and $\alpha_{k}=\frac{4}{k^{0.6}}$ in the distributed primal-dual method. The penalty parameter $r_{i}$ is 1.3 for all $i$ in algorithm (10)(11). The Bregman-distance generating functions are the Euclidean norms. Each algorithm is run for 4000 iterations.

Figure 2 shows the behavior of algorithms (10)-(11) and (37)-(38). As seen in the figure, both centralized algorithms perform the best (as they have the whole knowledge of the problem information) and they have a similar behavior. The distributed algorithms are slightly worse than the centralized, which is expected due to their "decentralized" incomplete knowledge of the problem. Of the two distributed algorithms, the primal-dual algorithm is worse, as it often assigns much larger allocations than the distributed exact penalty method. Primal-dual algorithms (in absence of strong convexity of the primal) are known to be highly sensitive to the choices of dual variables, which may be reflected in the results we see in Figure 2, where the primal-dual algorithms use the initial values $\mu_{i}=\frac{1}{m}$ for the multipliers. 


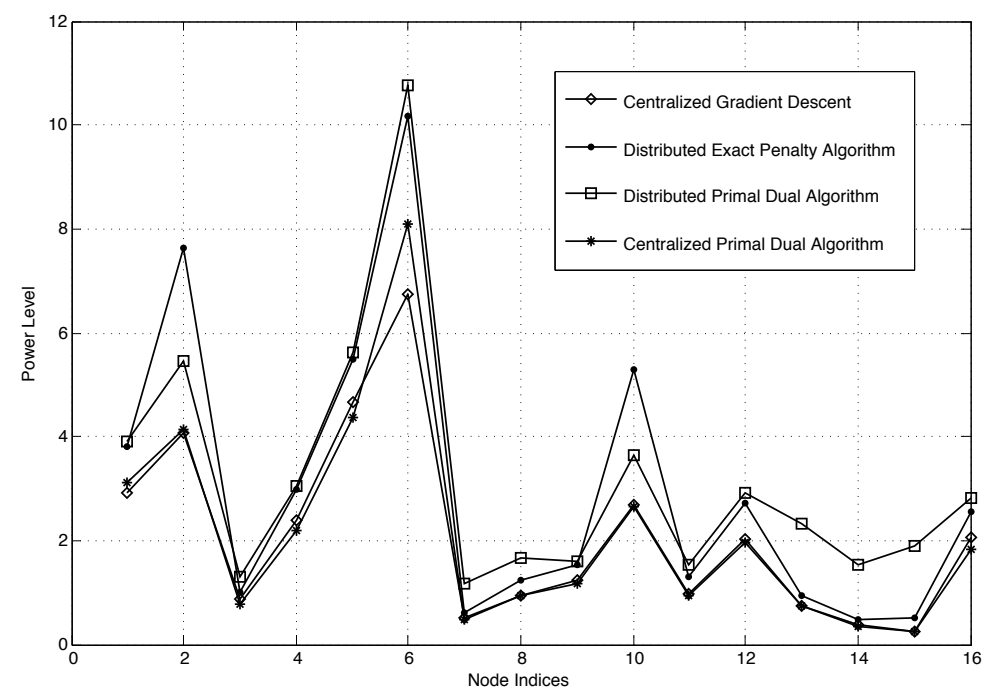

Fig. 2 The final iterate values after 4000 iterations of the algorithms. The plot shows the allocations achieved by Centralized Gradient Descent, Distributed Exact Penalty Algorithm, Distributed Primal-Dual Algorithm, and Centralized Primal-Dual Algorithm

\section{Conclusion}

We presented distributed algorithms for solving stochastic min-max optimization problems in networks. We developed two algorithms based on Bregman-distance functions. The first algorithm uses a non-differentiable penalty function to translate the min-max problem to a format which is suitable for distributed algorithms. The second algorithm is based on the primal-dual iterative update scheme. In both of these algorithms we allow the presence of stochastic subgradient noise. We provided conditions on the dynamic network under which we can guarantee almost sure convergent behavior of the algorithms. We illustrated the applicability of the algorithms on a power allocation problem in a cellular network.

The effectiveness of these algorithms is highly dependable on the underlying connectivity structure of the agent network. For future work, we plan to investigate error bounds for the proposed algorithms, which will capture the scalability of the algorithms with the number $m$ of agents. Based on our prior work [31], we know that these algorithms can scale at best in the order of $m^{3 / 2}$ when the sum of the functions $f_{i}$ is to be minimized and the $\sum_{i=1}^{m} f_{i}$ is strongly convex. We believe that this bound is also achievable by the proposed algorithms for a class of functions $f_{i}$, such as linear for example. Such results will also provide better insights into the practical short-term behavior of these algorithms.

Acknowledgements This work has been supported by the NSF Grant CMMI-0742538. 


\section{References}

1. A. Agarwal, J. Duchi, and M. Wainwright. Dual averaging for distributed optimization: Convergence analysis and network scaling. IEEE Transactions on Automatic Control, 2011. To appear.

2. K.J. Arrow, L. Hurwicz, and H. Uzawa. Studies in Linear and Non-Linear Programming. Stanford University Press, Stanford, CA, 1958.

3. D.P. Bertsekas. Necessary and sufficient conditions for a penalty method to be exact. Mathematical Programming, 9:87-99, 1975.

4. D.P. Bertsekas, A. Nedić, and A. Ozdaglar. Convex Analysis and Optimization. Athena Scientific, Belmont, MA, USA, 2003.

5. D.P. Bertsekas and J.N. Tsitsiklis. Parallel and distributed computation: numerical methods. Prentice-Hall, Inc., Upper Saddle River, NJ, USA, 1989.

6. D.P. Bertsekas and J.N. Tsitsiklis. Gradient convergence in gradient methods with errors. SIAM J. OPTIM., 10(3):627-642, 2000.

7. P. Billingsley. Probability and Measure. John Wiley and Sons, 1979.

8. H. Boche, M. Wiczanowski, and S. Stanczak. Unifying view on min-max fairness and utility optimization in cellular networks. In Wireless Communications and Networking Conference, 2005 IEEE, volume 3, pages 1280 - 1285 Vol. 3, march 2005.

9. V.S. Borkar. Stochastic Approximation: A Dynamical Systems Viewpoint. Cambridge University Press, 2008.

10. L. Bregman. The relaxation method of finding the common point of convex sets and its application to the solution of problems in convex programming. USSR Computational Mathematics and Mathematical Physics, 7(3):200-217, 1967.

11. Y. A. Censor and S.A. Zenios. Parallel Optimization: Theory, Algorithms and Applications. Oxford University Press, 1997.

12. M. Chiang, P. Hande, T. Lan, and W.C. Tan. Power Control in Wireless Cellular Networks. Found. Trends Netw., 2(4):381-533, 2008.

13. Y. Ermoliev. Stochastic quasi-gradient methods and their application to system optimization. Stochastics, 9(1):1-36, 1983

14. Y. Ermoliev. Stochastic quazigradient methods. In Numerical Techniques for Stochastic Optimization, pages 141-186. Springer-Verlag, N.Y., 1988.

15. T. Hastie, R. Tibshirani, and J. H. Friedman. The Elements of Statistical Learning. Springer, 2003.

16. A. Jadbabaie, J. Lin, and S. Morse. Coordination of groups of mobile autonomous agents using nearest neighbor rules. IEEE Transactions on Automatic Control, 48:988-1001, 2003.

17. B. Johansson, M. Rabi, and M. Johansson. A randomized incremental subgradient method for distributed optimization in networked systems. SIAM Journal on Optimization, 20(3):11571170, Aug 2009.

18. S. Kar and J.M.F. Moura. Distributed consensus algorithms in sensor networks with imperfect communication: link failures and channel noise. IEEE Tran. Signal Process., 57(1):355-369, 2009.

19. I. Lobel and A. Ozdaglar. Distributed subgradient methods for convex optimization over random networks. IEEE Transactions on Automatic Control, 56(6):1291-1306, june 2011.

20. D. Mosk-Aoyama, T. Roughgarden, and D. Shah. Fully distributed algorithms for convex optimization problems. In DISC '07: Proceedings of the 21st international symposium on Distributed Computing, pages 492-493, Berlin, Heidelberg, 2007. Springer-Verlag.

21. A. Nedić. Asynchronous broadcast-based convex optimization over a network. IEEE Transactions on Automatic Control, 56(6):1337-1351, 2011.

22. A. Nedić, A. Olshevsky, A. Ozdaglar, and J. N. Tsitsiklis. Distributed subgradient algorithms and quantization effects. In Proceedings of the 47th IEEE Conference on Decision and Control, pages 4177-4184, 2008

23. A. Nedić and A. Ozdaglar. Distributed subgradient methods for multi-agent optimization. IEEE Transactions on Automatic Control, 54(1):48-61, 2009. 
24. A. Nedić and A. Ozdaglar. Subgradient methods for saddle-point problems. Journal of Optimization Theory and Applications, 142(1):205-228, 2009.

25. A. Nedić, A. Ozdaglar, and P. A. Parrilo. Constrained consensus and optimization in multiagent networks. IEEE Transactions on Automatic Control, 55:922-938, 2010.

26. A. Nemirovski, A. Juditsky, G. Lan, and A. Shapiro. Robust stochastic approximation approach to stochastic programming. SIAM J. on Optimization, 19(4):1574-1609, 2008.

27. B. T. Polyak. Introduction to Optimization. Optimization Software, Inc., New York, 1987.

28. M. Rabbat and R. D. Nowak. Distributed optimization in sensor networks. In IPSN, pages 20-27, 2004.

29. S. S. Ram, A. Nedić, and V. V. Veeravalli. Distributed stochastic subgradient projection algorithms for convex optimization. Journal of Optimization Theory and Applications, 147(3):516-545, 2010.

30. S. S. Ram, V. V. Veeravalli, and A. Nedić. Distributed non-autonomous power control through distributed convex optimization. In IEEE INFOCOM, pages 3001-3005, 2009.

31. S. Sundhar Ram, A. Nedić, and V.V. Veeravalli. Asynchronous gossip algorithms for stochastic optimization: Constant stepsize analysis. In M. Diehl, F. Glineur, E. Jarlebring, and W. Michiels, editors, Recent Advances in Optimization and its Applications in Engineering, pages 51-60. Volume of the 14th Belgian-French-German Conference on Optimization (BFG), 2010.

32. S.S. Ram, A. Nedić, and V.V. Veeravalli. A new class of distributed optimization algorithms: Application to regression of distributed data. Optimization Methods and Software, 27(1):71$88,2012$.

33. H. Robbins and D. Siegmund. A convergence theorem for nonnegative almost supermartingales and some applications. In J.S. Rustagi, editor, Proceedings of a Symposium on Optimizing Methods in Statistics, pages 233-257. Academic Press, New York, 1971.

34. R. Srikant. The Mathematics of Internet Congestion Control. Birkhäuser, Boston, 2003.

35. K. Srivastava and A. Nedić. Distributed asynchronous constrained stochastic optimization. IEEE Journal of Selected Topics in Signal Processing, 5(4):772 -790, aug. 2011.

36. K. Srivastava, A. Nedić, and D. Stipanović. Distributed min-max optimization in networks. In 17th International Conference on Digital Signal Processing, 2011.

37. S.S. Stanković, M.S. Stanković, and D.M. Stipanović. Decentralized parameter estimation by consensus based stochastic approximation. In Decision and Control, 2007 46th IEEE Conference on, pages $1535-1540$, dec. 2007.

38. J. N. Tsitsiklis. Problems in decentralized decision making and computation. PhD thesis, Massachusetts Institute of Technology, Boston, 1984.

39. J. N. Tsitsiklis and M. Athans. Convergence and asymptotic agreement in distributed decision problems. IEEE Trans. Automat. Control, 29:42-50, 1984. 